JOURNAL OF THE

AMERICAN MATHEMATICAL SOCIETY

Volume 16, Number 2, Pages 363-392

S 0894-0347(02)00412-5

Article electronically published on November 29, 2002

\title{
TOTALLY POSITIVE TOEPLITZ MATRICES AND QUANTUM COHOMOLOGY OF PARTIAL FLAG VARIETIES
}

\author{
KONSTANZE RIETSCH
}

\section{INTRODUCTION}

A matrix is called totally nonnegative if all of its minors are nonnegative. Totally nonnegative infinite Toeplitz matrices were studied first in the 1950's. They are characterized in the following theorem conjectured by Schoenberg and proved by Edrei.

Theorem 1.1 ([10]). The $\infty \times \infty$ Toeplitz matrix

$$
A=\left(\begin{array}{cccccccc}
1 & & & & & & & \\
a_{1} & 1 & & & & & & \\
a_{2} & a_{1} & 1 & & & & & \\
\vdots & a_{2} & a_{1} & \ddots & & & & \\
a_{d} & & \ddots & \ddots & \ddots & & & \\
a_{d+1} & \ddots & & \ddots & a_{1} & 1 & & \\
\vdots & \ddots & \ddots & & a_{2} & a_{1} & \ddots & \\
& & \ddots & \ddots & & \ddots & \ddots & \ddots
\end{array}\right)
$$

is totally nonnegative precisely if its generating function is of the form,

$$
1+a_{1} t+a_{2} t^{2}+\cdots=\exp (t \alpha) \prod_{i \in \mathbb{N}} \frac{\left(1+\beta_{i} t\right)}{\left(1-\gamma_{i} t\right)},
$$

where $\alpha \in \mathbb{R}_{\geq 0}$ and $\beta_{1} \geq \beta_{2} \geq \cdots \geq 0, \gamma_{1} \geq \gamma_{2} \geq \cdots \geq 0$ with $\sum \beta_{i}+\sum \gamma_{i}<\infty$.

This beautiful result has been reproved many times; see 32 for an overview. It may be thought of as giving a parameterization of the totally nonnegative Toeplitz matrices by

$$
\left\{\left(\alpha ;\left(\tilde{\beta}_{i}\right)_{i},\left(\tilde{\gamma}_{i}\right)_{i}\right) \in \mathbb{R}_{\geq 0} \times \mathbb{R}_{\geq 0}^{\mathbb{N}} \times \mathbb{R}_{\geq 0}^{\mathbb{N}} \mid \sum_{i \in \mathbb{N}} i\left(\tilde{\beta}_{i}+\tilde{\gamma}_{i}\right)<\infty\right\},
$$

where $\tilde{\beta}_{i}=\beta_{i}-\beta_{i+1}$ and $\tilde{\gamma}_{i}=\gamma_{i}-\gamma_{i+1}$.

Received by the editors December 10, 2001 and, in revised form, September 14, 2002.

2000 Mathematics Subject Classification. Primary 20G20, 15A48, 14N35, 14N15.

Key words and phrases. Flag varieties, quantum cohomology, total positivity.

During a large part of this work the author was an EPSRC postdoctoral fellow (GR/M09506/01) and Fellow of Newnham College in Cambridge. The article was completed while supported by the Violette and Samuel Glasstone Foundation at Oxford.

(C)2002 American Mathematical Society 
Now let $U^{-}$denote the lower triangular unipotent $n \times n$ matrices. One aim of this paper is to parameterize the set of totally nonnegative matrices in

$$
X:=\left\{x \in U^{-} \mid x=\left(\begin{array}{cccccc}
1 & & & & & \\
a_{1} & 1 & & & & \\
a_{2} & a_{1} & 1 & & & \\
a_{3} & \ddots & \ddots & \ddots & & \\
\vdots & \ddots & \ddots & a_{1} & 1 & \\
a_{n-1} & \ldots & a_{3} & a_{2} & a_{1} & 1
\end{array}\right)\right\}
$$

by $n-1$ nonnegative parameters. Let $\Delta_{n-i}(x)$ be the lower left-hand corner $i \times i$ minor of $x \in X$. Explicitly, we will prove the following statement.

Proposition 1.2. Let $X_{\geq 0}$ denote the set of totally nonnegative matrices in $X$. Then the map

is a homeomorphism.

$$
\Delta_{\geq 0}:=\left(\Delta_{1}, \ldots, \Delta_{n-1}\right): X_{\geq 0} \longrightarrow \mathbb{R}_{\geq 0}^{n-1}
$$

Note that $\Delta: X \rightarrow \mathbb{C}^{n-1}$ is a ramified cover and the nonnegativity of the values of the $\Delta_{i}$ is by no means sufficient for an element $u$ to be totally nonnegative. The statement is rather that for prescribed nonnegative values of $\Delta_{1}, \ldots, \Delta_{n-1}$, among all matrices with these fixed values there is precisely one which is totally nonnegative.

The proof of this result involves relating total positivity for these $n \times n$ Toeplitz matrices to properties of quantum cohomology rings of partial flag varieties, via Dale Peterson's realization of these as coordinate rings of certain remarkable subvarieties of the flag variety. We show that the Schubert basis of the quantum cohomology ring plays a similar role for these matrices with regard to positivity as does the (classical limit of the) dual canonical basis for the whole of $U^{-}$in the work of Lusztig [24]. This is the content of Theorem [7.2, which is the main result of this paper. The above parameterization of $X_{\geq 0}$ comes as a corollary.

1.1. Overview of the paper. The first part of the paper is taken up with introducing the machinery we will need to prove our results. We set out by recalling background on the quantum cohomology rings of full and partial flag varieties, especially work of Astashkevich, Sadov, Kim, and Ciocan-Fontanine, as well as Fomin, Gelfand and Postnikov.

Their work is then used in Section 4 to explain Peterson's result identifying these rings as coordinate rings of affine strata of a certain remarkable subvariety $\mathcal{Y}$ of the flag variety. The variety $X$ of Toeplitz matrices enters the picture when the Peterson variety $\mathcal{Y}$ is viewed from the opposite angle $\left(U^{-}\right.$-orbits rather than $U^{+}$orbits). We recall the Bruhat decomposition of the variety of Toeplitz matrices. Each stratum $X_{P}$ has in its coordinate ring the quantum cohomology ring of a partial flag variety $G / P$ with its Schubert basis and quantum parameters.

In Section 5 we recall Kostant's formula for the quantum parameters as functions on $X_{B}$ in terms of the minors $\Delta_{i}$ and generalize it to the partial flag variety case.

After some motivation from total positivity the main results are stated in Section [7. Theorem [7.2 has three parts. Firstly, the set of points in $X_{P}$ where all Schubert basis elements take positive values has a parameterization $\left(q_{1}, \ldots, q_{k}\right)$ : $X_{P,>0} \stackrel{\sim}{\longrightarrow} \mathbb{R}_{>0}^{k}$ given by the quantum parameters. Secondly, this set lies in the 
smooth locus of $X_{P}$ and the inverse of the map giving the parameterization is analytic. Thirdly, this set of Schubert positive points agrees with the set of totally nonnegative matrices in $X_{P}$. Proposition [1.2 stated in the introduction is proved immediately as a corollary.

In Section 8 we make an excursion to recall what these results look like explicitly in the Grassmannian case, which is studied in detail in an earlier paper. We then use the Grassmannian components of the Peterson variety to prove that the top Schubert class $\sigma_{w_{0} P}$ is generically nonvanishing as function on $X_{P}$. Conjecturally, the same should hold for the quantum Euler class, $\sum_{w \in W^{P}} \sigma_{w} \sigma_{P D(w)}$, which would imply that $q H^{*}(G / P)$ is reduced.

The rest of the paper is devoted to the proof of Theorem 7.2. In the next two sections, parts (1) and (2) of the main theorem are proved. The main ingredient for constructing and parameterizing the Schubert positive points is the positivity of the structure constants (Gromov-Witten invariants). Computing the fiber in $X_{P}$ over a fixed positive value of the quantum parameters $\left(q_{1}, \ldots, q_{k}\right)$ is turned into an eigenvalue problem for an irreducible nonnegative matrix, and the unique Schubert positive solution we require is provided by a Perron-Frobenius eigenvector. The smoothness property turns out to be related to the positivity of the quantum Euler class, while bianalyticity comes as a consequence of the one-dimensionality of the Perron-Frobenius eigenspace.

The final part of Theorem 7.2, that the notion of positivity coming from Schubert bases agrees with total positivity, is perhaps the most surprising. The problem is that, except in the case of Grassmannian permutations, we know no useful way to compute the Schubert classes as functions on the $X_{P}$. In Section [11 we begin to simplify this problem by proving another remarkable component of Peterson's theory. Namely, consider the functions on $X_{B}$ given by the Schubert classes of $q H^{*}(G / B)$. Then when extended as rational functions to all of $X$, these restrict to give all the Schubert classes on the smaller strata $X_{P}$. We prove this explicitly using quantum Schubert polynomials and Fomin, Gelfand and Postnikov's quantum straightening identity.

This last result enables us essentially to reduce the proof of the final part of Theorem 7.2 to the full flag variety case. The main problem there is to prove that an arbitrary Schubert class takes positive values on the totally positive part. This is done by topological arguments, using that the totally positive part of $X_{B}$ is a semigroup.

\section{Preliminaries}

Let $G=G L_{n}(\mathbb{C})$, and $I=\{1, \ldots, n-1\}$ an indexing set for the simple roots. Denote by Ad the adjoint representation of $G$ on its Lie algebra $\mathfrak{g}$. We fix the Borel subgroups $B^{+}$of upper-triangular matrices and $B^{-}$of lower-triangular matrices in $G$. Their Lie algebras are denoted by $\mathfrak{b}^{+}$and $\mathfrak{b}^{-}$, respectively. We will also consider their unipotent radicals $U^{+}$and $U^{-}$with their Lie algebras $\mathfrak{u}^{+}$and $\mathfrak{u}^{-}$and the maximal torus $T=B^{+} \cap B^{-}$. Let $X^{*}(T)$ be the character group of $T$ and $X_{*}(T)$ the group of cocharacters with the usual perfect pairing $<,>: X^{*}(T) \times X_{*}(T) \rightarrow \mathbb{Z}$ between them. Let $\Delta_{+} \subset X^{*}(T)$ be the set of positive roots corresponding to $\mathfrak{b}^{+}$, and $\Delta_{-}$the set of negative roots. The fundamental weights and coweights are denoted by $\omega_{1}, \ldots, \omega_{n-1} \in X^{*}(T)$ and $\omega_{1}^{\vee}, \ldots, \omega_{n-1}^{\vee} \in X_{*}(T)$, respectively. We define $\Pi \subset X^{*}(T)$ to be the set of positive simple roots. The elements of $\Pi$ are 
denoted $\alpha_{1}, \ldots, \alpha_{n-1}$ where the $\alpha_{m}$-root space $\mathfrak{g}_{\alpha_{m}} \in \mathfrak{g}$ is spanned by

$$
e_{\alpha_{m}}=\left(\delta_{i}^{m} \delta_{j}^{m+1}\right)_{i, j=1}^{n}=\left(\begin{array}{cccccc}
0 & & & & & \\
& \ddots & & & & \\
& & 0 & 1 & & \\
& & & 0 & & \\
& & & & \ddots & \\
& & & & & 0
\end{array}\right) \text {. }
$$

Let $e:=\sum_{m=1}^{n-1} e_{\alpha_{m}}$. A special role will be played by the principal nilpotent element $f \in \mathfrak{u}^{-}$which is the transpose of $e$.

We identify the Weyl group $W$ of $G$, the symmetric group, with the group of all permutation matrices. $W$ is generated by the usual simple reflections (adjacent transpositions) $s_{1}, \ldots, s_{n-1}$. The length function $\ell: W \rightarrow \mathbb{N}$ gives the length of a reduced expression of $w \in W$ in the simple generators. There is a unique longest element which is denoted $w_{0}$.

2.1. Parabolics. Let $P$ always denote a parabolic subgroup of $G$ containing $B^{-}$, and $\mathfrak{p}$ the Lie algebra of $P$. Let $I_{P}$ be the subset of $I$ associated to $P$ consisting of all the $i \in I$ with $s_{i} \in P$ and consider its complement $I^{P}:=I \backslash I_{P}$. We will denote the elements of $I^{P}$ by $\left\{n_{1}, \ldots, n_{k}\right\}$ with

$$
n_{0}:=0<n_{1}<n_{2}<\cdots<n_{k}<n_{k+1}:=n .
$$

Then the homogeneous space $G / P$ may be identified with the partial flag variety (of quotients)

$$
G / P=\mathcal{F}_{n_{1}, \ldots, n_{k}}\left(\mathbb{C}^{n}\right)=\left\{\mathbb{C}^{n} \rightarrow V_{k} \rightarrow \cdots \rightarrow V_{1} \rightarrow 0 \mid \operatorname{dim} V_{j}=n_{j}\right\} .
$$

Next introduce $W_{P}=\left\langle s_{i} \mid i \in I_{P}\right\rangle$, the parabolic subgroup of $W$ corresponding to $P$, and $W^{P} \subset W$, the set of minimal coset representatives for $W / W_{P}$. An element $w$ lies in $W^{P}$ precisely if for all reduced expressions $w=s_{i_{1}} \cdots s_{i_{m}}$ the last index $i_{m}$ always lies in $I^{P}$. We write $w^{P}$ or $w_{0}^{P}$ for the longest element in $W^{P}$, while the longest element in $W_{P}$ is denoted $w_{P}$. For example $w_{0}^{B}=w_{0}$ and $w_{B}=1$. Finally $P$ gives rise to a decomposition

$$
\Delta_{+}=\Delta_{P,+} \sqcup \Delta_{+}^{P},
$$

where $\Delta_{P,+}=\left\{\alpha \in \Delta_{+} \mid\left\langle\alpha, \omega_{i}^{\vee}\right\rangle=0\right.$ for all $\left.i \in I^{P}\right\}$ and $\Delta_{+}^{P}$ is its complement. So, for example, $\Delta_{B,+}=\emptyset$ and $\Delta_{+}^{B}=\Delta_{+}$.

\section{The Quantum Cohomology Ring of $G / P$}

3.1. The usual cohomology of $G / P$ and its Schubert basis. For our purposes it will suffice to take homology or cohomology with complex coefficients, so always $H^{*}(G / P)=H^{*}(G / P, \mathbb{C})$. By the well-known result of $\mathrm{C}$. Ehresmann, the singular homology of the partial flag variety $G / P$ has a basis indexed by the elements $w \in W^{P}$ made up of the fundamental classes of the Schubert varieties,

$$
\Omega_{w}^{P}:=\overline{\left(B^{+} w P / P\right)} \subseteq G / P .
$$

Here the bar stands for (Zariski) closure. Let $\sigma_{w}^{P} \in H^{*}(G / P)$ be the Poincaré dual class to $\left[\Omega_{w}^{P}\right]$. Note that $\Omega_{w}^{P}$ has complex codimension $\ell(w)$ in $G / P$ and hence $\sigma_{w}^{P}$ lies in $H^{2 \ell(w)}(G / P)$. The set $\left\{\sigma_{w}^{P} \mid w \in W^{P}\right\}$ forms a basis of $H^{*}(G / P)$ called 
the Schubert basis. The top degree cohomology of $G / P$ is spanned by $\sigma_{w_{0}^{P}}^{P}$ and we have the Poincaré duality pairing

$$
H^{*}(G / P) \times H^{*}(G / P) \longrightarrow \mathbb{C}, \quad(\sigma, \mu) \mapsto\langle\sigma \cdot \mu\rangle
$$

which may be interpreted as taking $(\sigma, \mu)$ to the coefficient of $\sigma_{w_{0}^{P}}^{P}$ in the basis expansion of the product $\sigma \cdot \mu$. For $w \in W^{P}$ let $P D(w) \in W^{P}$ be the minimal length coset representative in $w_{0} w W_{P}$. Then this pairing is characterized by

$$
\left\langle\sigma_{w} \cdot \sigma_{v}\right\rangle=\delta_{w, P D(v)} .
$$

3.2. Definition of the quantum cohomology ring $q H^{*}(G / P)$. The (small) quantum cohomology ring $q H^{*}(G / P)$ may be defined by enumerating curves into $G / P$ with certain properties. This description is responsible for its positivity properties and is the one we will give here. For more general background there are already many books and survey articles on the subject of quantum cohomology; see e.g. [9, 13, 29, 30] and references therein.

Let $I^{P}=\left\{n_{1}, \ldots, n_{k}\right\}$. Then as a vector space the quantum cohomology of the partial flag variety $G / P$ is given by

$$
q H^{*}(G / P)=\mathbb{C}\left[q_{1}^{P}, \ldots, q_{k}^{P}\right] \otimes_{\mathbb{C}} H^{*}(G / P),
$$

where $q_{1}^{P}, \ldots, q_{k}^{P}$ are called the quantum parameters. Consider the Schubert classes as elements of $q H^{*}(G / P)$ by identifying $\sigma_{w}^{P}$ with $1 \otimes \sigma_{w}^{P}$. We will sometimes drop the superscript $P$ from the notation for the Schubert classes and the quantum parameters when there is no possible ambiguity.

Now $q H^{*}(G / P)$ is a free $\mathbb{C}\left[q_{1}^{P}, \ldots, q_{k}^{P}\right]$-module with basis given by the Schubert classes $\sigma_{w}^{P}$. It remains to give the structure constants $\left\langle\sigma_{u}^{P}, \sigma_{v}^{P}, \sigma_{w}^{P}\right\rangle_{\mathbf{d}}$ in

$$
\sigma_{v}^{P} \sigma_{w}^{P}=\sum_{\substack{u \in W^{P} \\ \mathbf{d} \in \mathbb{N}^{k}}}\left\langle\sigma_{u}^{P}, \sigma_{v}^{P}, \sigma_{w}^{P}\right\rangle_{\mathbf{d}} \mathbf{q}^{\mathbf{d}} \sigma_{P D(u)}^{P}
$$

to define the ring structure on $q H^{*}(G / P)$. Here $\mathbf{q}^{\mathbf{d}}$ is multi-index notation for $\prod_{i=1}^{k} q_{i}^{d_{i}}$.

Consider the set $\mathcal{M}_{\mathbf{d}}$ of holomorphic maps $\phi: \mathbb{C P}^{1} \rightarrow G / P$ such that

$$
\phi_{*}\left(\left[\mathbb{C P}^{1}\right]\right)=\sum_{i=1}^{k} d_{i}\left[\overline{B^{-} s_{n_{i}} P / P}\right] .
$$

$\mathcal{M}_{\mathbf{d}}$ can be made into a quasi-projective variety of dimension equal to $\operatorname{dim}(G / P)+$ $\sum d_{i}\left(n_{i+1}-n_{i-1}\right)$. To define $\left\langle\sigma_{u}^{P}, \sigma_{v}^{P}, \sigma_{w}^{P}\right\rangle_{\mathbf{d}}$ first translate the Schubert varieties $\Omega_{u}^{P}, \Omega_{v}^{P}$ and $\Omega_{w}^{P}$ into general position, say to $\widetilde{\Omega_{u}^{P}}, \widetilde{\Omega_{v}^{P}}$ and $\widetilde{\Omega_{w}^{P}}$. Now consider the set $\mathcal{M}_{\mathbf{d}}(u, v, w)$ of all maps $\phi \in \mathcal{M}_{\mathbf{d}}$ such that

$$
\phi(0) \in \widetilde{\Omega_{u}^{P}}, \quad \phi(1) \in \widetilde{\Omega_{v}^{P}}, \quad \text { and } \quad \phi(\infty) \in \widetilde{\Omega_{w}^{P}} .
$$

Then $\mathcal{M}_{\mathbf{d}}(u, v, w)$ is finite if $\operatorname{dim}(G / P)+\sum d_{i}\left(n_{i+1}-n_{i-1}\right)=\ell(u)+\ell(v)+\ell(w)$ and one may set

$$
\left\langle\sigma_{u}^{P}, \sigma_{v}^{P}, \sigma_{w}^{P}\right\rangle_{\mathbf{d}}=\left\{\begin{array}{ll}
\# \mathcal{M}_{\mathbf{d}}(u, v, w) & \text { if } \operatorname{dim}(G / P)+\sum d_{i}\left(n_{i+1}-n_{i-1}\right) \\
0 & \text { otherwise. }
\end{array}=\ell(u)+\ell(v)+\ell(w),\right.
$$


These quantities are 3-point, genus 0 Gromov-Witten invariants. By looking at $\mathbf{d}=$ $(0, \ldots, 0)$ one recovers the classical structure constants obtained from intersecting Schubert varieties in general position. Therefore this multiplicative structure is a deformation of the classical cup product. We note that the structure constants by their definition are nonnegative integers.

The quantum cohomology analogue of the Poincaré duality pairing may be defined as the symmetric $\mathbb{C}\left[q_{1}^{P}, \ldots, q_{k}^{P}\right]$-bilinear pairing

$$
q H^{*}(G / P) \times q H^{*}(G / P) \longrightarrow \mathbb{C}\left[q_{1}^{P}, \ldots, q_{k}^{P}\right], \quad(\sigma, \mu) \mapsto\langle\sigma \cdot \mu\rangle_{\mathbf{q}}
$$

which takes $(\sigma, \mu)$ to the coefficient of $\sigma_{w_{0}^{P}}^{P}$ in the Schubert basis expansion of the product $\sigma \cdot \mu$. In terms of the Schubert basis the quantum Poincaré duality pairing on $q H^{*}(G / P)$ is given by

$$
\left\langle\sigma_{w}^{P} \cdot \sigma_{v}^{P}\right\rangle_{\mathbf{q}}=\delta_{w, P D(v)}
$$

where $v, w \in W^{P}$, and $P D: W^{P} \rightarrow W^{P}$ is the involution defined in Section 3.1 (see e.g. [8], Lemma 6.1).

3.3. Borel's presentation of the cohomology ring $H^{*}(G / P)$. Let $G / P$ be realized as a variety of flags of quotients as in Section 2.1.

$$
G / P=\mathcal{F}_{n_{1}, \ldots, n_{k}}\left(\mathbb{C}^{n}\right)=\left\{\mathbb{C}^{n}=V_{k+1} \rightarrow V_{k} \rightarrow \cdots \rightarrow V_{1} \rightarrow 0 \mid \operatorname{dim} V_{j}=n_{j}\right\} .
$$

Then for $1 \leq j \leq k+1$, the successive quotients $Q_{j}=\operatorname{ker}\left(V_{j} \rightarrow V_{j-1}\right)$ define rank $\left(n_{j}-n_{j-1}\right)$ vector bundles on $G / P$. Their Chern classes will be denoted

$$
c_{i}\left(Q_{j}\right)=: \sigma_{i}^{(j)}=\sigma_{i, P}^{(j)}
$$

By the splitting principle it is natural to introduce independent variables $x_{1}, \ldots, x_{n}$ such that $x_{n_{j-1}+1}, \ldots, x_{n_{j}}$ are the Chern roots of $Q_{j}$. So

$$
\sigma_{i}^{(j)}=e_{i}\left(x_{n_{j-1}+1}, x_{n_{j-1}+2}, \ldots, x_{n_{j}}\right),
$$

the $i$-th elementary symmetric polynomial in the variables $\left\{x_{n_{i}+1}, \ldots, x_{n_{i+1}}\right\}$. Let $W_{P}$ act on the polynomial ring $\mathbb{C}\left[x_{1}, \ldots, x_{n}\right]$ in the natural way by permuting the variables. Then the ring of invariants is

$$
\mathbb{C}\left[x_{1}, \ldots, x_{n}\right]^{W_{P}}=\mathbb{C}\left[\sigma_{1}^{(1)}, \ldots, \sigma_{n_{1}}^{(1)}, \sigma_{1}^{(2)}, \ldots \ldots, \sigma_{1}^{(k+1)}, \ldots, \sigma_{n-n_{k}}^{(k+1)}\right] .
$$

A. Borel [5] showed that the Chern classes $\sigma_{i}^{(j)}$ generate $H^{*}(G / P)$ and the only relations between these generators come from the triviality of the bundle $Q_{1} \oplus Q_{2} \oplus$ $\cdots \oplus Q_{k}$ (which may be trivialized using a Hermitian inner product on $\mathbb{C}^{n}$ ).

In other words, if $J$ denotes the ideal in $\mathbb{C}\left[x_{1}, \ldots, x_{n}\right]^{W_{P}}$ generated by the elementary symmetric polynomials $e_{i}\left(x_{1}, \ldots, x_{n}\right)$, then

$$
H^{*}(G / P) \cong \mathbb{C}\left[x_{1}, \ldots, x_{n}\right]^{W_{P}} / J .
$$

3.4. Schubert polynomials and elementary monomials for $H^{*}(G / P)$. For $1 \leq i \leq k+1$, define

$$
e_{i}^{(j)}=e_{i, P}^{(j)}:=e_{i}\left(x_{1}, \ldots, x_{n_{j}}\right),
$$

the $i$-th elementary symmetric polynomial in $n_{j}$ variables. Then the $e_{i}^{(k+1)}$ 's are the generators of the ideal $J$. But for $1 \leq j \leq k$, the element $e_{i}^{(j)}$ corresponds 
under (3.2) to a nonzero element of $H^{*}(G / P)$, namely the special Schubert class $\sigma_{s_{n_{j}-i+1} \cdots s_{n_{j}}}^{P}$.

The polynomial defined by

$$
c_{w_{0}^{P}}:=\left(e_{n_{1}}^{(1)}\right)^{n_{1}} \cdots\left(e_{n_{k}}^{(k)}\right)^{n_{k}-n_{k-1}}
$$

represents the top class $\sigma_{w_{0}^{P}}$.

These are examples of the Schubert polynomials of Lascoux and Schützenberger, 23. The Schubert polynomials $\left\{c_{w} \mid w \in W^{P}\right\} \subset \mathbb{C}\left[x_{1}, \ldots, x_{n}\right]^{W_{P}}$ are, loosely speaking, obtained from the top one by divided difference operators; see [23] or [27] for details. If $P=B$ then Schubert polynomials $c_{w}$ are obtained for all $w \in W$, and the ones from above corresponding to $G / P$ are just the subset consisting of all those for which $w \in W^{P}$. The key property of a Schubert polynomial $c_{w}$ is of course that it is a representative for the corresponding Schubert class $\sigma_{w}$.

A different description, following [11, of the Schubert polynomials $c_{w}$ for $w \in$ $W^{P}$ says precisely where these representatives must lie. They are those representatives of the Schubert classes which may be written as linear combinations of certain "elementary monomials" in $\mathbb{C}\left[x_{1}, \ldots, x_{n}\right]^{W_{P}}$.

Explicitly, let $\mathcal{L}_{P}$ be the set of sequences $\Lambda=\left(\lambda^{(1)}, \ldots, \lambda^{(k)}\right)$ of partitions, such that $\lambda^{(j)}$ has at most $\left(n_{j}-n_{j-1}\right)$ parts and $\lambda_{1}^{(j)} \leq n_{j}$. To any $\Lambda \in \mathcal{L}_{P}$ associate a polynomial,

$$
e_{\Lambda}=\left(e_{\lambda_{1}^{(1)}}^{(1)} \cdots e_{\lambda_{n_{1}}^{(1)}}^{(1)}\right) \cdots\left(e_{\lambda_{1}^{(k)}}^{(k)} \cdots e_{\lambda_{n_{k}-n_{k-1}}^{(k)}}^{(k)}\right) .
$$

Let us call these polynomials $P$-standard monomials. These $e_{\Lambda}$ are linearly independent and span a complementary subspace to the ideal $J$. So

$$
\mathbb{C}\left[x_{1}, \ldots, x_{n}\right]^{W_{P}}=J \oplus\left\langle e_{\Lambda}\right\rangle_{\Lambda \in \mathcal{L}_{P}} .
$$

Then the Schubert polynomial $c_{w}$ is the (unique) representative in $\left\langle e_{\Lambda}\right\rangle_{\Lambda \in \mathcal{L}_{P}}$ for the Schubert class $\sigma_{w}^{P}$.

3.5. Astashkevich, Sadov and Kim's presentation of $q H^{*}(G / P)$. The presentation for the quantum cohomology ring $q H^{*}(G / P)$ analogous to Borel's presentation of $H^{*}(G / P)$ was first discovered by Astashkevich and Sadov [2] and Kim 18. A complete proof may be found in Ciocan-Fontanine 8]. In special cases these presentations were known earlier, e.g. for Grassmannians [4, 36], and in the full flag variety case [16, 7].

The generators of $q H^{*}(G / P)$ will be the generators of the usual cohomology ring $\sigma_{i}^{(j)}$ (embedded as $1 \otimes \sigma_{i}^{(j)}$ ) along with the quantum parameters $q_{1}^{P}, \ldots, q_{k}^{P}$. Here $i, j$ runs through $1 \leq j \leq k+1$ and $1 \leq i \leq n_{j}-n_{j-1}$. Let us for now treat the $\sigma_{i}^{(j)}$ and $q_{j}$ as independent variables generating a polynomial ring $\mathbb{C}\left[\sigma_{1}^{(1)}, \ldots, \sigma_{n-n_{k}}^{(k+1)}, q_{1}, \ldots, q_{k}\right]$.

Definition 3.1 ((q,P)-elementary symmetric polynomials). Let $i \in \mathbb{Z}$ and $l \in$ $\{-1,0, \ldots, k+1\}$. Define elements $E_{i, P}^{(l)}=E_{i}^{(l)} \in \mathbb{C}\left[\sigma_{1}^{(1)}, \ldots, \sigma_{n-n_{k}}^{(k+1)}, q_{1}, \ldots, q_{k}\right]$ recursively as follows. The initial values are

$$
E_{i}^{(-1)}=E_{i}^{(0)}=0 \text { for all } i \text {, and } E_{i}^{(l)}=0 \text { unless } 0 \leq i \leq n_{l},
$$


and we set $\sigma_{i}^{(l)}=0$ if $i>n_{l}-n_{l-1}$ and $\sigma_{0}^{(l)}=1$. For $1 \leq l \leq k+1$ and $0 \leq i \leq n_{l}$ the polynomial $E_{i}^{(l)}$ is defined by

$$
\begin{aligned}
E_{i}^{(l)}=\left(E_{i}^{(l-1)}+E_{i-1}^{(l-1)} \sigma_{1}^{(l)}+\cdots+E_{1}^{(l-1)} \sigma_{i-1}^{(l)}\right. & \left.+\sigma_{i}^{(l)}\right) \\
& +(-1)^{n_{l}-n_{l-1}+1} q_{l-1} E_{i-n_{l}+n_{l-2}}^{(l-2)} .
\end{aligned}
$$

If the $q_{l}$ are set to 0 and the $\sigma_{i}^{(l)}$ are as in (3.1), then this recursion defines the elementary symmetric polynomials $e_{i}^{(l)}$.

Remark 3.2. This is a basic recursive definition of the quantum elementary symmetric polynomials. See [8] for a host of other descriptions. And here is also one other curious one to add to this list.

Order the variables $\sigma_{i}^{(j)}$ lexicographically, so that $\sigma_{i}^{(j)}<\sigma_{k}^{\left(j^{\prime}\right)}$ whenever $j<j^{\prime}$ or $j=j^{\prime}$ and $i<k$. Now suppose just for the remainder of this remark that the variables $\sigma_{i}^{(j)}$ are not necessarily commuting. More precisely, let $\sigma_{i}^{(j)}$ and $\sigma_{k}^{\left(j^{\prime}\right)}$ commute unless $\left|j-j^{\prime}\right|=1$ and both $i$ and $k$ are maximal. In that case impose the Heisenberg relation

$$
\sigma_{n_{j+1}-n_{j}}^{(j+1)} \sigma_{n_{j}-n_{j-1}}^{(j)}=\sigma_{n_{j}-n_{j-1}}^{(j)} \sigma_{n_{j+1}-n_{j}}^{(j+1)}+(-1)^{n_{j+1}-n_{j}+1} q_{j}
$$

The $q_{i}$ commute with everything. Now add a central variable $x$ and define polynomials $p_{j}(x)=x^{n_{j}-n_{j-1}}+\sigma_{1}^{(j)} x^{n_{j}-n_{j-1}-1}+\cdots+\sigma_{n_{j}-n_{j-1}}^{(j)}$. Then expand the product

$$
p_{m}(x) \cdot p_{m-1}(x) \cdot \cdots \cdot p_{1}(x)
$$

and write the resulting coefficients in terms of increasing monomials in the $\sigma_{i}^{(j)}$ (monomials with factors ordered in increasing fashion) by using the commutation relations. Then for $d \leq n_{m}$ the coefficient of $x^{n_{m}-d}$ gives the $(\mathbf{q}, P)$-elementary symmetric polynomial $E_{d}^{(m)}$.

For example, in type $A_{2}$ for the full flag variety case, the polynomials $E_{1}^{(3)}, E_{2}^{(3)}$ and $E_{3}^{(3)}$ turn up as coefficients in

$$
\begin{aligned}
&\left(x+x_{3}\right)\left(x+x_{2}\right)\left(x+x_{1}\right)=x^{3}+\left(x_{1}+x_{2}+x_{3}\right) x^{2} \\
&+\left(x_{1} x_{2}+x_{2} x_{3}+x_{1} x_{3}+q_{1}+q_{2}\right) x+\left(x_{1} x_{2} x_{3}+x_{1} q_{2}+x_{3} q_{1}\right) .
\end{aligned}
$$

Theorem $3.3([\underline{8},[2], 18])$. The assignment $\sigma_{j}^{(i)} \mapsto 1 \otimes \sigma_{j}^{(i)}$ and $q_{i} \mapsto q_{i} \otimes 1$ gives rise to an isomorphism

$$
\mathbb{C}\left[\sigma_{1}^{(1)}, \ldots, \sigma_{n-n_{k}}^{(k)}, q_{1}, \ldots, q_{k}\right] / J \stackrel{\sim}{\longrightarrow} q H^{*}(G / P),
$$

where $J$ is the ideal $\left(E_{1}^{(k+1)}, \ldots, E_{n}^{(k+1)}\right)$. This isomorphism takes the element $E_{i}^{(l)}$ for $1 \leq l \leq k$ to the special Schubert class $\sigma_{s_{n_{l}-i+1} \cdots s_{n_{l}}}^{P}$.

An immediate question raised by this theorem is how to describe Schubert classes in the picture on the left-hand side of this isomorphism. This is answered by a quantum analogue of the Schubert polynomials. 
3.6. Quantum Schubert polynomials. In the case of $G / B$ a full theory of quantum Schubert polynomials was given by Fomin, Gelfand and Postnikov [1]. This was later generalized to partial flag varieties by Ciocan-Fontanine [8]. Note that the quantum Schubert polynomials for partial flag varieties are not special cases of the full flag variety ones, due to lack of functoriality of quantum cohomology (but see Proposition 11.1).

There is also a different construction of (double) quantum Schubert polynomials due to Kirillov and Maeno [20] which has been shown to give the same answer. We give the definitions following [11] and [8].

Definition $3.4((\mathbf{q}, P)$-standard monomials $)$. As in Section [3.4, let $\mathcal{L}_{P}$ be the set of sequences $\Lambda=\left(\lambda^{(1)}, \ldots, \lambda^{(k)}\right)$ of partitions such that $\lambda^{(j)}$ has at most $\left(n_{j}-n_{j-1}\right)$ parts and $\lambda_{1}^{(j)} \leq n_{j}$. To each $\Lambda \in \mathcal{L}_{P}$ associate an element

$$
E_{\Lambda}=\left(E_{\lambda_{1}^{(1)}}^{(1)} \cdots E_{\lambda_{n_{1}}^{(1)}}^{(1)}\right) \cdots\left(E_{\lambda_{1}^{(k)}}^{(k)} \cdots E_{\lambda_{n_{k}-n_{k-1}}^{(k)}}^{(k)}\right) \in \mathbb{C}\left[\sigma_{1}^{(1)}, \ldots, \sigma_{n-n_{k}}^{(k)}, q_{1}, \ldots, q_{k}\right] .
$$

These elements are called the $(\mathbf{q}, P)$-standard monomials.

Remark 3.5 (Example). The $(\mathbf{q}, B)$-standard polynomials were introduced in [11]. They are the monomials of the form

$$
E_{B, j_{1}}^{(1)} E_{B, j_{2}}^{(2)} \cdots E_{B, j_{n-1}}^{(n-1)}
$$

where $0 \leq j_{l} \leq l$ for all $l=1, \ldots, n-1$.

Let $V$ denote the $\mathbb{C}\left[q_{1}, \ldots, q_{k}\right]$-module spanned by the $(\mathbf{q}, P)$-standard monomials,

$$
V=\mathbb{C}\left[q_{1}, \ldots, q_{k}\right] \otimes_{\mathbb{C}}\left\langle E_{\Lambda}\right\rangle_{\Lambda \in \mathcal{L}_{P}} .
$$

Then

$$
\mathbb{C}\left[\sigma_{1}^{(1)}, \ldots, \sigma_{n-n_{k}}^{(k)}, q_{1}, \ldots, q_{k}\right]=J \oplus V .
$$

Definition 3.6 ((q,P)-Schubert polynomials). The quantum Schubert polynomial $C_{w}^{P} \in \mathbb{C}\left[\sigma_{1}^{(1)}, \ldots, \sigma_{n-n_{k}}^{(k+1)}, q_{1}, \ldots, q_{k}\right]$ is defined to be the unique element of $V$ whose coset modulo $J$ maps to the Schubert class $\sigma_{w}^{P}$ under the isomorphism

$$
\mathbb{C}\left[\sigma_{1}^{(1)}, \ldots, \sigma_{n-n_{k}}^{(k+1)}, q_{1}, \ldots, q_{k}\right] / J \stackrel{\sim}{\longrightarrow} q H^{*}(G / P) .
$$

Remark 3.7 (Example). From (3.4) it follows immediately that the (q, $P)$-Schubert polynomial representing the top class $\sigma_{w_{0}^{P}} \in q H^{*}(G / P)$ is given by

$$
C_{w_{0}^{P}}^{P}=\left(E_{P, n_{1}}^{(1)}\right)^{n_{1}} \cdots\left(E_{P, n_{k}}^{(k)}\right)^{n_{k}-n_{k-1}} .
$$

3.7. Grassmannian permutations. A Grassmannian permutation of descent $m$ is an element $w \in W^{P_{d}}$ for the maximal parabolic $P_{d}$ with $I^{P_{d}}=\{d\}$. As permutations on $\{1, \ldots, n\}$ these may be characterized by

$$
w \in W^{P_{d}} \Longleftrightarrow w(1)<\cdots<w(d) \text { and } w(d+1)<\cdots<w(n) .
$$

They are in bijective correspondence with shapes (partitions) $\lambda=\left(\lambda_{1}, \ldots, \lambda_{d}\right)$ such that $n-d \geq \lambda_{1} \geq \cdots \geq \lambda_{d} \geq 0$, via $\lambda_{i}=w(i)-i+1$.

Let $w_{\lambda, d}$ denote the Grassmannian permutation of descent $d$ and shape $\lambda$. There is a closed formula for the quantum Schubert polynomials $C_{w_{\lambda, d}}^{B}$ given by A. N. Kirillov in [19], which we will derive here from Fomin, Gelfand and Postnikov's definition. 
The classical Schubert polynomial for $w_{\lambda, d}$ is just the Schur polynomial $c_{w_{\lambda, d}}=$ $s_{\lambda}\left(x_{1}, \ldots, x_{d}\right)$; see e.g. [27]. Therefore by the Jacobi-Trudi identity

$$
c_{w_{\lambda, d}}=\operatorname{det}\left(\begin{array}{cccc}
e_{\lambda_{1}^{\prime}}^{(d)} & e_{\lambda_{1}^{\prime}+1}^{(d)} & \cdots & e_{\lambda_{1}^{\prime}+c-1}^{(d)} \\
e_{\lambda_{2}^{\prime}-1}^{(d)} & e_{\lambda_{2}^{\prime}}^{(d)} & \cdots & e_{\lambda_{2}^{\prime}+c-2}^{(d)} \\
& & \ddots & \\
e_{\lambda_{c}^{\prime}+c-1}^{(d)} & \cdots & \cdots & e_{\lambda_{c}^{\prime}}^{(d)}
\end{array}\right)
$$

where $\lambda^{\prime}$ is the conjugate partition to $\lambda$ and $c=n-d$ (see [28]). Repeatedly applying the identity

$$
e_{j}^{(m)}=e_{j}^{(m+1)}-x_{m+1} e_{j-1}^{(m)}
$$

of elementary symmetric polynomials to one column in the determinant at a time, one obtains

$$
c_{w_{\lambda, d}}=\operatorname{det}\left(\begin{array}{cccc}
e_{\lambda_{1}^{\prime}}^{(d)} & e_{\lambda_{1}^{\prime}+1}^{(d+1)} & \cdots & e_{\lambda_{1}^{\prime}+c-1}^{(n-1)} \\
e_{\lambda_{2}^{\prime}-1}^{(d)} & e_{\lambda_{2}^{\prime}}^{(d+1)} & \cdots & e_{\lambda_{2}^{\prime}+c-2}^{(n-1)} \\
& & \ddots & \\
e_{\lambda_{c}^{\prime}+c-1}^{(d)} & \cdots & \cdots & e_{\lambda_{c}^{\prime}}^{(n-1)}
\end{array}\right) .
$$

Expanding out this determinant gives an expression for $c_{w_{\lambda, d}}$ as linear combination of $B$-standard monomials. Therefore the quantization is simply given by

$$
C_{w_{\lambda, d}}=\operatorname{det}\left(\begin{array}{cccc}
E_{\lambda_{1}^{\prime}}^{(d)} & E_{\lambda_{1}^{\prime}+1}^{(d+1)} & \cdots & E_{\lambda_{1}^{\prime}+c-1}^{(n-1)} \\
E_{\lambda_{2}^{\prime}-1}^{(d)} & E_{\lambda_{2}^{\prime}}^{(d+1)} & \cdots & E_{\lambda_{2}^{\prime}+c-2}^{(n-1)} \\
& & \ddots & \\
E_{\lambda_{c}^{\prime}+c-1}^{(d)} & \cdots & \cdots & E_{\lambda_{c}^{\prime}}^{(n-1)}
\end{array}\right) .
$$

3.8. Quantum Chevalley formula. The Pieri formula for $H^{*}(G / P)$ of Lascoux and Schützenberger was generalized to the $q H^{*}(G / P)$ setting by Ciocan-Fontanine in [8]. We will only need the following simpler case.

Theorem 3.8. For $h \leq l \in\{1, \ldots, k\}$ set $\tau_{h, l}=s_{n_{h}} \cdot \ldots \cdot s_{n_{l+1}-1} s_{n_{l}-1} \cdot \ldots$. $s_{n_{h-1}+1}$ and $\mathbf{q}_{h, l}=q_{h} \cdot q_{h+1} \cdot \cdots \cdot q_{l}$. Let $n_{j} \in I^{P}$ and $w \in W^{P}$. Then

$$
\sigma_{s_{n_{j}}}^{P} \sigma_{w}^{P}=\sum_{\substack{\alpha \in \Delta^{+} \\
w s_{\alpha} \in W^{P} \\
\ell\left(w s_{\alpha}\right)=\ell(w)+1}}<\alpha, \omega_{n_{j}}^{\vee}>\sigma_{w s_{\alpha}}^{P}+\sum_{\begin{array}{c}
h, l \in\{1, \ldots, k\} \\
1 \leq h \leq j \leq l \leq k \\
\ell\left(w \tau_{h, l}\right)=\ell(w)-\ell\left(\tau_{h, l}\right)
\end{array}} \mathbf{q}_{h, l} \sigma_{w \tau_{h, l}}^{P}
$$

This is a reformulation of a special case of Theorem 3.1 in [8].

\section{QUANTUM COHOMOLOGY RINGS AS COORDINATE RINGS}

4.1. ASK-matrices. We define with some minor changes an $n \times n$ matrix $A^{[k+1]}$ with entries in $\mathbb{C}\left[\sigma_{1}^{(1)}, \ldots, \sigma_{n-n_{k}}^{(k)}, q_{1}, \ldots, q_{k}\right]$ introduced by Astashkevich, Sadov and $\mathrm{Kim}$ in [2] and [18]. Setting $n_{k+1}=n$ and $n_{0}=0$ define first $\left(n_{j}-n_{j-1}\right) \times$ 
$\left(n_{j}-n_{j-1}\right)$ matrices $D^{(j)}$ by

$$
D^{(1)}=\left(\begin{array}{cccc}
-\sigma_{1}^{(1)} & -\sigma_{2}^{(1)} & \ldots & -\sigma_{n_{1}}^{(1)} \\
0 & \cdots & \ldots & 0 \\
\vdots & & & \vdots \\
\vdots & & & \vdots \\
0 & \ldots & \ldots & 0
\end{array}\right) \text { and } D^{(j)}=\left(\begin{array}{cccc}
0 & \ldots & 0 & -\sigma_{n_{j}-n_{j-1}}^{(j)} \\
\vdots & & \vdots & \vdots \\
\vdots & & \vdots & -\sigma_{2}^{(j)} \\
0 & \ldots & 0 & -\sigma_{1}^{(j)}
\end{array}\right)
$$

for $2 \leq j \leq k+1$, and let $D^{[l]}$ be the $n_{l} \times n_{l}$ block matrix made up of diagonal blocks $D^{(1)}, \ldots, D^{(l)}$. Furthermore define $n_{l} \times n_{l}$ matrices

$$
f^{[l]}=\left(\begin{array}{cccc}
0 & & & \\
1 & \ddots & & \\
& \ddots & \ddots & \\
& & 1 & 0
\end{array}\right) \text { and } Q^{[l]}:=\left((-1)^{n_{m+1}-n_{m}} q_{m} \delta_{i}^{n_{m-1}+1} \delta_{j}^{n_{m+1}}\right)_{i, j=1}^{n_{l}} .
$$

Then set

$$
A^{[l]}:=f^{[l]}+D^{[l]}+Q^{[l]} .
$$

The coefficients of the characteristic polynomials of the $A^{[l]}$ satisfy precisely the same recursion as the $(P, \mathbf{q})$-standard symmetric polynomials $E_{P, i}^{(l)}$. Explicitly, we have

$$
\operatorname{det}\left(\lambda \operatorname{Id}-A^{[l]}\right)=\lambda^{n_{l}}+E_{P, 1}^{(l)} \lambda^{n_{l}-1}+\cdots+E_{P, n_{l}}^{(l)} .
$$

In particular the relations $E_{1}^{(k+1)}=\cdots=E_{n}^{(k+1)}=0$ of the quantum cohomology ring are equivalent to

$$
\operatorname{det}\left(\lambda \operatorname{Id}-A^{[k+1]}\right)=\lambda^{n} .
$$

Let us call the matrices in $\mathfrak{g l}_{n}$ of the same form as $A^{[k+1]}$ (with the same pattern of 0 and 1 entries) $A S K$-matrices. They form an affine subspace $\mathcal{A}_{P}$ in $\mathfrak{g l}_{n}$. Let $\mathcal{N}_{P}$ be the (nonreduced) intersection,

$$
\mathcal{N}_{P}=\mathcal{A}_{P} \cap \mathcal{N},
$$

of $\mathcal{A}_{P}$ with the nilpotent cone $\mathcal{N}$ in $\mathfrak{g l}_{n}$. Its coordinate ring is denoted $\mathcal{O}\left(\mathcal{N}_{P}\right)$.

Then (4.1) implies that the map $\mathcal{O}\left(\mathcal{A}_{P}\right) \longrightarrow \mathbb{C}\left[\sigma_{1}^{(1)}, \ldots, \sigma_{n-n_{k}}^{(k)}, q_{1}, \ldots, q_{k}\right]$ defined by $A^{[k+1]}$ induces an isomorphism

$$
\mathcal{O}\left(\mathcal{N}_{P}\right) \stackrel{\sim}{\longrightarrow} \mathbb{C}\left[\sigma_{1}^{(1)}, \ldots, \sigma_{n-n_{k}}^{(k)}, q_{1}, \ldots, q_{k}\right] / J
$$

The statement of Theorem 3.3 may therefore be interpreted as

$$
\mathcal{O}\left(\mathcal{N}_{P}\right) \stackrel{\sim}{\longrightarrow} q H^{*}(G / P) .
$$

4.2. Peterson's theorem. All the affine varieties $\operatorname{Spec}\left(q H^{*}(G / P)\right)$ turn out to be most naturally viewed as embedded in the flag variety (or in general in the Langlands dual flag variety) where they patch together as strata of one remarkable projective variety called the Peterson variety. This is the content of Dale Peterson's theorem which we will deduce here explicitly for the type $A$ case.

Definition 4.1. Let $\mathfrak{b}^{++}:=\sum_{\alpha \in \Delta_{+} \backslash \Pi} \mathfrak{g}_{\alpha}$ and $\pi^{++}: \mathfrak{g} \rightarrow \mathfrak{b}^{++}$be the projection along weight spaces. Let $f \in \mathfrak{g l}_{n}$ be the principal nilpotent $f^{[k+1]}$ from above. Then the equations

$$
\pi^{++}\left(\operatorname{Ad}\left(g^{-1}\right) \cdot f\right)=0
$$


define a closed subvariety of $G$ invariant under right multiplication by $B^{-}$. Thus they define a closed subvariety of $G / B^{-}$. This subvariety $\mathcal{Y}$ is the Peterson variety for type $A$. Loosely, $\mathcal{Y}$ can be described by

$$
\mathcal{Y}=\left\{g B^{-} \in G / B^{-} \mid \operatorname{Ad}\left(g^{-1}\right) \cdot f \in \mathfrak{b}^{-} \oplus \sum_{i \in I} \mathbb{C} e_{\alpha_{i}}\right\} .
$$

Let $v_{1}, \ldots, v_{n}$ be the standard basis of $V=\mathbb{C}^{n}$. Then $\left\{v_{i_{1}} \wedge \cdots \wedge v_{i_{j}} \mid 0<\right.$ $\left.i_{1}<i_{2}<\cdots<i_{j}<n\right\}$ is the standard basis of the fundamental representation $V^{\omega_{j}}=\bigwedge^{j} V$. Let $(\mid)$ denote the inner product on $V^{\omega_{j}}$ such that this basis is orthonormal. We also refer to representations by their lowest weight, so $V^{\omega_{n-m}}=$ : $V_{-\omega_{m}}$. Denote the lowest weight vector by

$$
v_{-\omega_{m}}=v_{m+1} \wedge \cdots \wedge v_{n}
$$

Define rational functions $G_{i}^{m}=G_{s_{m-i+1} \cdots s_{m}}$ on $G / B^{-}$in terms of matrix coefficients of the fundamental representations by

$$
G_{i}^{m}\left(g B^{-}\right)=G_{s_{m-i+1} \cdots s_{m}}\left(g B^{-}\right):=\frac{\left(g \cdot v_{-\omega_{m}} \mid s_{m-i+1} \cdots s_{m} \cdot v_{-\omega_{m}}\right)}{\left(g \cdot v_{-\omega_{m}} \mid v_{-\omega_{m}}\right)}
$$

Note that $s_{m-i+1} \cdots s_{m} \cdot v_{-\omega_{m}}=v_{m-i+1} \wedge v_{m+2} \wedge \cdots \wedge v_{n}$ and $G_{i}^{m}\left(g B^{-}\right)$may be written down simply as a quotient of two $(n-m) \times(n-m)$ minors of $g$.

Theorem 4.2 (D. Peterson). (1) For any parabolic subgroup $W_{P} \subset W$ with longest element $w_{P}$ define $\mathcal{Y}_{P}$ as (nonreduced) intersection by

$$
\mathcal{Y}_{P}:=\mathcal{Y} \cap\left(B^{+} w_{P} B^{-} / B^{-}\right) \text {. }
$$

Then on points these give a decomposition

$$
\mathcal{Y}(\mathbb{C})=\bigsqcup_{P} \mathcal{Y}_{P}(\mathbb{C})
$$

(2) For each parabolic $P$ there is a unique isomorphism

$$
\mathcal{O}\left(\mathcal{Y}_{P}\right) \stackrel{\sim}{\longrightarrow} q H^{*}(G / P),
$$

which sends $G_{s_{n_{j}-i+1} \cdots s_{n_{j}}}$ to $\sigma_{s_{n_{j}-i+1} \cdots s_{n_{j}}}^{P}$.

Remark 4.3. If $P$ is the parabolic subgroup, then $G_{j}^{m}$ is a well-defined (regular) function on the Bruhat cell $B^{+} w_{P} B^{-} / B^{-}$precisely in the case $m \in I^{P}=$ $\left\{n_{1}, \ldots, n_{k}\right\}$. In fact we have

$$
\begin{aligned}
& B^{+} w_{P} B^{-} / B^{-} \stackrel{\sim}{\longrightarrow} \mathbb{C}\left(\sum_{i=1}^{k} n_{i}\right) \\
& g B^{-} \mapsto\left(G_{1}^{n_{1}}\left(g B^{-}\right), \ldots, G_{n_{1}}^{n_{1}}\left(g B^{-}\right), G_{1}^{n_{2}}\left(g B^{-}\right), \ldots, G_{n_{k}}^{n_{k}}\left(g B^{-}\right)\right),
\end{aligned}
$$

or in other words,

$$
\mathcal{O}\left(B^{+} w_{P} B^{-} / B^{-}\right)=\mathbb{C}\left[G_{1}^{n_{1}}, \ldots, G_{n_{k}}^{n_{k}}\right] .
$$

Let $\mathcal{J}_{P} \subset \mathbb{C}\left[G_{1}^{n_{1}}, \ldots, G_{n_{k}}^{n_{k}}\right]$ denote the ideal defining $\mathcal{Y}_{P}$.

Proof. (1) is proved in [33]. See also Lemma 2.3 in [35]. We will deduce (2) very explicitly from the ASK presentation. Begin by defining a particular section 
$u: B^{+} w_{P} B^{-} / B^{-} \rightarrow U^{+}$of the map $x \mapsto x w_{P} B^{-}$in the other direction. For $l=0, \ldots, k$ we have $n \times\left(n_{l+1}-n_{l}\right)$ matrices $U^{(l)}$ defined by

$$
U^{(0)}=\left(\begin{array}{ccccc}
1 & G_{1}^{n_{1}} & G_{2}^{n_{1}} & \cdots & G_{n_{1}-1}^{n_{1}} \\
0 & 1 & G_{1}^{n_{1}} & \ddots & \vdots \\
\vdots & & \ddots & & G_{2}^{n_{1}} \\
& & & \ddots & G_{1}^{n_{1}} \\
& & & & 0 \\
& & & & \vdots \\
& & & & \\
\vdots & & & & \vdots \\
0 & \ldots & & \ldots & 0
\end{array}\right), \quad U^{(l)}=\left(\begin{array}{ccccc}
G_{n_{l}}^{n_{l}} & & & \\
\vdots & \ddots & & \\
\vdots & & \ddots & \\
G_{2}^{n_{l}} & & & G_{n_{l}}^{n_{l}} \\
G_{1}^{n_{l}} & \ddots & & \vdots \\
1 & \ddots & \ddots & \vdots \\
0 & \ddots & G_{1}^{n_{l}} & G_{2}^{n_{l}} \\
\vdots & & 1 & G_{1}^{n_{l}} \\
& & & & 1 \\
\vdots & & & \\
0 & \ldots & \ldots & 0
\end{array}\right)
$$

where $1 \leq l \leq k$. Then the matrix

$$
u=\left(\begin{array}{l|l|l|l} 
& & & \\
U^{(0)} & U^{(1)} & \ldots & U^{(k)} \\
& & &
\end{array}\right)
$$

defines a map $u: B^{+} w_{P} B^{-} / B^{-} \rightarrow U^{+}$. It follows using Remark 4.3 that this map is indeed a section. That is,

$$
g B^{-}=u\left(g B^{-}\right) w_{P} B^{-}, \quad \text { for } g B^{-} \in B^{+} w_{P} B^{-} / B^{-} .
$$

Consider the matrix

$$
\tilde{A}=u^{-1} f u \in \mathfrak{g l}_{n}\left(\mathbb{C}\left[G_{1}^{n_{1}}, \ldots, G_{n_{k}}^{n_{k}}\right]\right) .
$$

A direct computation shows that modulo the ideal $\mathcal{J}_{P}$ defining $\mathcal{Y}_{P}$ the matrix $\tilde{A}$ is an ASK matrix (i.e. it is an ASK matrix over $\mathbb{C}\left[G_{1}^{n_{1}}, \ldots, G_{n_{k}}^{n_{k}}\right] / \mathcal{J}_{P}$ ). Also it is clear that the characteristic polynomial of $\tilde{A}$ satisfies $\operatorname{det}(\lambda \operatorname{Id}-\tilde{A})=\lambda^{n}$, since $\tilde{A}$ is conjugate to $f$. Therefore the morphism $B^{+} w_{P} B^{-} / B^{-} \longrightarrow \mathfrak{g l}_{\mathfrak{n}}$ defined by $\tilde{A}$ restricts to a morphism

$$
\left.\tilde{A}\right|_{\mathcal{Y}_{P}}: \mathcal{Y}_{P} \rightarrow \mathcal{N}_{P}
$$

from $\mathcal{Y}_{P}$ to the variety of nilpotent ASK-matrices.

For the inverse define a map $\psi: \mathbb{C}\left[G_{1}^{n_{1}}, \ldots, G_{n_{k}}^{n_{k}}\right] \rightarrow \mathbb{C}\left[\sigma_{1}^{(1)}, \ldots, \sigma_{n-n_{k}}^{(k+1)}, q_{1}, \ldots, q_{k}\right]$ by $\psi\left(G_{i}^{n_{j}}\right)=E_{i}^{(j)}$. Applying $\psi$ to the entries of $u$ we obtain a matrix $u_{E}$ with entries in $\mathbb{C}\left[\sigma_{1}^{(1)}, \ldots, \sigma_{n-n_{k}}^{(k+1)}, q_{1}, \ldots, q_{k}\right]$. Then the recursive definition of the $E_{i}^{(j)}$ translates into the identity

$$
u_{E}^{-1} f u_{E}=A^{[k+1]}+M
$$


of matrices over $\mathbb{C}\left[\sigma_{1}^{(1)}, \ldots, \sigma_{n-n_{k}}^{(k+1)}, q_{1}, \ldots, q_{k}\right]$, where $A^{[k+1]}$ is the matrix defined in Section 4.1 and $M$ the $n \times n$ matrix given by

$$
M=\left(\begin{array}{ccccc}
0 & \cdots & \cdots & 0 & E_{n}^{(k+1)} \\
\vdots & & & \vdots & E_{n-1}^{(k+1)} \\
\vdots & & & \vdots & \vdots \\
\vdots & & & \vdots & E_{2}^{(k+1)} \\
0 & \cdots & \cdots & 0 & E_{1}^{(k+1)}
\end{array}\right) .
$$

This identity implies that $\psi$ induces a map of quotient rings

$$
\tilde{\psi}: \mathcal{O}\left(\mathcal{Y}_{P}\right) \longrightarrow \mathbb{C}\left[\sigma_{1}^{(1)}, \ldots, \sigma_{n-n_{k}}^{(k+1)}, q_{1}, \ldots, q_{k}\right] /\left(E_{1}^{(k+1)}, \ldots, E_{n}^{(k+1)}\right) .
$$

This map together with (4.2) defines an inverse $\mathcal{N}_{P} \rightarrow \mathcal{Y}_{P}$ to (4.6). Thus $\tilde{\psi}$ is an isomorphism and everything follows from Theorem 3.3 .

Remark 4.4. Actually, Peterson's results are more generally stated over the integers. And here in particular the analogous theorem over $\mathbb{Z}$ holds, with the exact same proof. We have stayed over $\mathbb{C}$ in our presentation since that is all we will require.

4.3. Toeplitz matrices. The stabilizer of $f$ under conjugation by $U^{-}$is precisely the $(n-1)$-dimensional abelian subgroup of lower-triangular unipotent Toeplitz matrices,

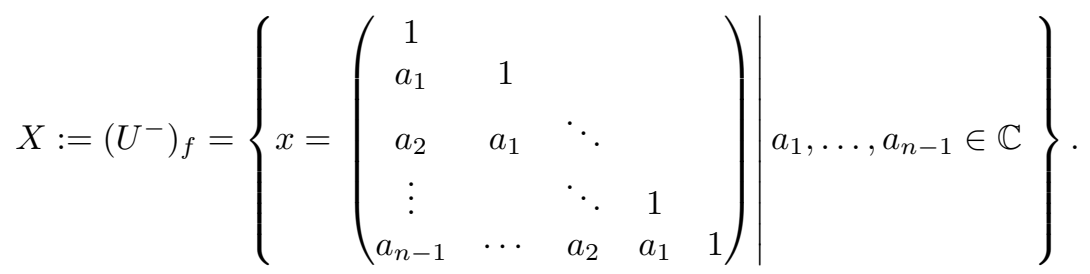

Let us take the matrix entries $a_{1}, \ldots, a_{n-1}$ as coordinates on $X$, thereby identifying $\mathcal{O}(X)=\mathbb{C}\left[a_{1}, \ldots, a_{n-1}\right]$. For $1 \leq m \leq n-1$ let $\Delta_{m} \in \mathcal{O}(X)$ be defined by

$$
\Delta_{m}=\operatorname{det}\left(a_{j-i+m}\right)_{i, j=1}^{n-m}=\left|\begin{array}{cccc}
a_{m} & a_{m-1} & \cdots & \\
a_{m+1} & \ddots & \ddots & \vdots \\
\vdots & \ddots & \ddots & a_{m-1} \\
a_{n-1} & \cdots & a_{m+1} & a_{m}
\end{array}\right|,
$$

where $a_{0}=1$ and $a_{l}=0$ if $l<0$. Let

$$
X_{P}=X \cap B^{+} w_{P} w_{0} B^{+} .
$$

We recall the following explicit description of the $X_{P} \subset X$.

Lemma 4.5 ([35] Lemma 2.5). As a subset (not subvariety) of $X, X_{P}$ is described by

$$
X_{P}=\left\{u \in X \mid \Delta_{i}(u) \neq 0 \Longleftrightarrow i \in\left\{n_{1}, \ldots, n_{k}\right\}\right\}
$$


Note that the map

$$
\Delta=\left(\Delta_{1}, \ldots, \Delta_{n-1}\right): X \rightarrow \mathbb{C}^{n-1}
$$

has the property $\Delta^{-1}(0)=\{0\}$ (in fact over any field). And $\Delta^{*}: \mathcal{O}\left(\mathbb{C}^{n-1}\right)=$ $\mathbb{C}\left[z_{1}, \ldots, z_{n-1}\right] \rightarrow \mathbb{C}\left[a_{1}, \ldots, a_{n-1}\right]$ is homogeneous, if the generators are taken with suitable degrees. Therefore $\Delta$ is a finite morphism; see e.g. [17].

Theorem 4.6 (D. Peterson). $\quad$ (1) Define

$$
\mathcal{X}:=\mathcal{Y} \cap\left(B^{-} w_{0} B^{-} / B^{-}\right) \quad \text { and } \quad \mathcal{X}_{P}:=\mathcal{X} \cap \mathcal{Y}^{P} .
$$

Then the isomorphism $U^{-} \rightarrow B^{-} w_{0} B^{-} / B^{-}$defined by $u \mapsto u w_{0} B^{-}$identifies $X$ with $\mathcal{X}$ and also $X_{P}$ with $\mathcal{X}_{P}$ for each parabolic $P$.

(2) The map 4.5 induces an isomorphism of $\mathcal{O}\left(\mathcal{X}_{P}\right)$ with $q H^{*}(G / P)\left[q_{1}^{-1}\right.$, $\left.\ldots, q_{k}^{-1}\right]$ giving

$$
\mathcal{O}\left(X_{P}\right) \stackrel{\sim}{\sim} \mathcal{O}\left(\mathcal{X}_{P}\right) \stackrel{\sim}{\longrightarrow} q H^{*}(G / P)\left[q_{1}^{-1}, \ldots, q_{k}^{-1}\right] .
$$

In particular, each $\mathcal{X}_{P}$ is open dense in $\mathcal{Y}_{P}$.

For a proof of this when $P=B$ see Theorems 8 and 9 in [21]. The general case is analogous, and for (2) see also the proof of Lemma 5.1 below.

\section{The Quantum Parameters as FUnCtions on $X_{P}$}

After applying (4.9), the quantum parameters $q_{j}^{P}$ may be expressed (up to taking some roots) in terms of the functions $\Delta_{i}$ from (4.8). In the full flag variety case, that is on $X_{B}$ where all $\Delta_{i}$ are nonvanishing, Kostant 21] has given the following formula:

$$
q_{j}^{B}=\frac{\Delta_{j-1} \Delta_{j+1}}{\left(\Delta_{j}\right)^{2}} .
$$

This generalizes as follows to the partial flag variety case.

Lemma 5.1. Let the quantum parameters $q_{j}^{P}$ be regarded as functions on $X_{P}$ via the isomorphism $\mathcal{O}\left(X_{P}\right) \cong q H^{*}(G / P)\left[q_{1}^{-1}, \ldots, q_{k}^{-1}\right]$ from (4.9). Then

$$
\left(q_{j}^{P}\right)^{\left(n_{j}-n_{j-1}\right)\left(n_{j+1}-n_{j}\right)}=\frac{\left(\Delta_{n_{j-1}}\right)^{n_{j+1}-n_{j}}\left(\Delta_{n_{j+1}}\right)^{n_{j}-n_{j-1}}}{\left(\Delta_{n_{j}}\right)^{n_{j+1}-n_{j-1}}} .
$$

The proof of this lemma which we give below is an adaptation of Kostant's proof of the formula (5.1).

Proof. Let $y \in X_{P}$. Then $y w_{0} B^{-} \in \mathcal{X}_{P}$ and we have

$$
y w_{0} B^{-}=u w_{P} B^{-} \quad \text { for some } u \in U^{+} \text {. }
$$

Without loss of generality $u$ may be chosen such that $\operatorname{Ad}\left(u^{-1}\right) \cdot f$ is an ASK-matrix $A \in \mathcal{N}_{P}$. Since $u w_{P} B^{-}=y w_{0} B^{-}$, we can find $\bar{u} \in U^{+}$and $t \in T$ such that

$$
y=u w_{P}^{-1} w_{0}^{-1} t \bar{u}^{-1} .
$$

We have

$$
\operatorname{Ad}\left(\bar{u}^{-1}\right) \cdot f=\operatorname{Ad}\left(t w_{0} w_{P}^{-1} u^{-1} y\right) \cdot f=\operatorname{Ad}\left(t w_{0} w_{P}^{-1}\right) \cdot A .
$$

The right-hand side may be expanded to give

$$
\operatorname{Ad}\left(t^{-1} w_{0} w_{P}\right) \cdot A=\sum_{i=1}^{n-1} m_{i} f_{i}+\text { higher weight space terms, }
$$


where explicitly

$$
m_{i}= \begin{cases}(-1)^{n_{j+1}-n_{j}} \alpha_{i}(t) q_{j}^{P}(y) & \text { if } i=n-n_{j}, \\ \alpha_{i}(t) & \text { if } n-i \notin\left\{n_{1}, \ldots, n_{k}\right\} .\end{cases}
$$

This follows from the isomorphisms in Section 4. On the other hand since $\bar{u} \in U^{+}$, the left-hand side of (5.2) implies that all the $m_{i}$ must be equal to 1 . Therefore we have the identities

$$
\begin{aligned}
\alpha_{n-n_{j}}(t) & =(-1)^{n_{j+1}-n_{j}} q_{j}^{P}(y)^{-1} & & j=1, \ldots, k, \\
\alpha_{i}(t) & =1 & & n-i \notin\left\{n_{1}, \ldots, n_{k}\right\} .
\end{aligned}
$$

Thus $t$ is determined up to a scalar factor $\lambda$ by the $q_{i}(y)$ 's. Let $T_{k+1}$ be the $\left(n-n_{k}\right) \times\left(n-n_{k}\right)$ identity matrix and $T_{j}$ the $\left(n_{j}-n_{j-1}\right) \times\left(n_{j}-n_{j-1}\right)$ matrix

$$
T_{j}=(-1)^{n-n_{j}} q_{j}(y) q_{j+1}(y) \cdots q_{k}(y)\left(\begin{array}{ccc}
1 & & \\
& \ddots & \\
& & 1
\end{array}\right),
$$

for $1 \leq j \leq k$. Then $t$ may explicitly be described by

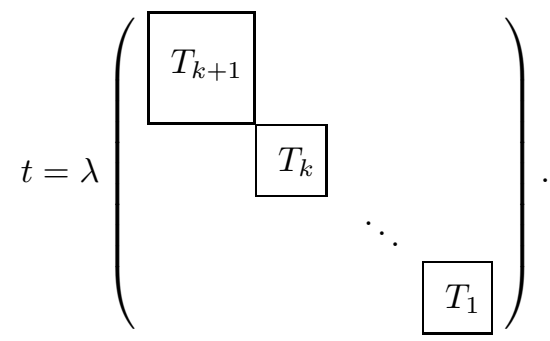

Now

$$
\begin{aligned}
\Delta_{n_{j}}(y) & =\left(y \cdot v_{1} \wedge \cdots \wedge v_{n-n_{j}+1} \mid v_{n_{j}+1} \wedge \cdots \wedge v_{n}\right) \\
& =\left(u w_{P}^{-1} w_{0}^{-1} t \bar{u}^{-1} \cdot v_{1} \wedge \cdots \wedge v_{n-n_{j}} \mid v_{n_{j}+1} \wedge \cdots \wedge v_{n}\right) \\
& =\omega_{n-n_{j}}(t)\left(u w_{P}^{-1} w_{0}^{-1} \cdot v_{1} \wedge \cdots \wedge v_{n-n_{j}} \mid v_{n_{j}+1} \wedge \cdots \wedge v_{n}\right) \\
& =\lambda^{n-n_{j}} q_{k}(y)^{n_{k}-n_{j}} q_{k-1}(y)^{n_{k-1}-n_{j}} \cdots q_{j+1}(y)^{n_{j+1}-n_{j}}
\end{aligned}
$$

and the identity follows.

\section{Total Positivity}

A matrix $A$ in $G L_{n}(\mathbb{R})$ is called totally positive (or totally nonnegative) if all the minors of $A$ are positive (respectively nonnegative). In other words $A$ acts by positive or nonnegative matrices in all the fundamental representations $\Lambda^{k} \mathbb{R}^{n}$ (with respect to their standard bases). These matrices clearly form a semigroup. The concept of totally positive matrices is in this sense more fundamental than the naive concept simply of matrices with positive entries, which overemphasizes the standard representation. Total positivity for $G L_{n}$ was mainly studied in and around the 1950's by Schoenberg, Gantmacher-Krein, Karlin and others, and has relationships with diverse applications such as oscillating mechanical systems and planar Markov processes.

More recently, G. Lusztig [24] extended the theory of total positivity to all reductive algebraic groups. His extension rests around a beautiful connection with 
canonical bases (for ADE type). This point of view on total positivity was part of the motivation for the main result of this paper, stated in the next section. It goes as follows.

Let us consider the lower uni-triangular matrices $U^{-}$(staying with $G=G L_{n}$, to avoid making further definitions). Let $U_{\geq 0}^{-}$be the set of totally nonnegative matrices in $U^{-}$. And define the "totally positive" part of $U^{-}$by

$$
U_{>0}^{-}:=U_{\geq 0}^{-} \cap B^{+} w_{0} B^{+} \text {. }
$$

Now the canonical basis of the quantized universal enveloping algebra $\mathcal{U}_{q}^{-}$defined by Lusztig and Kashiwara gives rise, after dualizing and taking the classical limit, to a basis $\mathcal{B}$ of the coordinate ring $\mathcal{O}\left(U^{-}\right)$. Lusztig proved that the canonical basis has positive structure constants for multiplication and comultiplication, using his geometric construction of $\mathcal{U}_{q}^{-}$. This is the main ingredient for the following theorem.

Theorem 6.1 ([25]; see also Section 3.13 in [26]). Suppose $u \in U^{-}(\mathbb{R})$. Then

$$
u \in U_{>0}^{-} \quad \Longleftrightarrow \quad b(u)>0 \quad \text { for all } b \in \mathcal{B} .
$$

The functions $b \in \mathcal{B}$ are matrix coefficients of $U^{-}$in irreducible representations of $G L_{n}$ with respect to canonical bases of these representations (obtained from the canonical basis of $\mathcal{U}_{q}^{-}$). This theorem is a reformulation of a result from [25], which holds for any simply laced reductive algebraic group.

Philosophically, $U^{-}$is a variety with a special basis on its coordinate ring (even a $\mathbb{Z}$-basis if we were to define $U^{-}$over the integers) which moreover has nonnegative integer structure constants. And by Lusztig's theorem, the question regarding which $u \in U^{-}$have the property that all $b \in \mathcal{B}$ are positive on $u$ has a very nice answer, namely the totally positive part of $U^{-}$.

We ask the same question for the components $\mathcal{Y}_{P}$ of the Peterson variety (or equivalently for the $X_{P} \subset U^{-}$), whose coordinate rings are naturally endowed with Schubert bases also with positive structure constants (as enumerative GromovWitten invariants). And remarkably we discover total positivity again in the answer.

The corollary, the parameterization result for totally nonnegative finite Toeplitz matrices stated in the introduction, also illustrates a common feature in total positivity. For example there are natural parameterizations of $U_{>0}^{-}$(introduced in [24]) which are related to combinatorics of the canonical basis and have been studied extensively. See e.g. 12 for a survey.

\section{Statement of the Main theOREM}

The varieties we are studying lie either inside $G L_{n}$ or $G / B^{-}$. By their real points we mean coming from their split real form, $G L_{n}(\mathbb{R})$ and the real flag variety. We consider the real points always to be endowed with the usual Hausdorff topology coming from $\mathbb{R}$. The positive parts will be semi-algebraic subsets of the real points.

Following [24], the totally positive part $\left(G / B^{-}\right)_{>0}$ of $G / B^{-}$is defined as the image of $U_{>0}^{+}$under the quotient map $G \rightarrow G / B^{-}$. By a result in [24] this agrees with the image of $U_{>0}^{-} w_{0} B^{-}$. So

$$
\left(G / B^{-}\right)_{>0}=U_{>0}^{+} B^{-} / B^{-}=U_{>0}^{-} w_{0} B^{-} / B^{-} .
$$

The totally nonnegative part $\left(G / B^{-}\right)_{\geq 0}$ is the closure of $\left(G / B^{-}\right)_{>0}$ inside the real flag variety. 
Using Peterson's isomorphisms (4.5) and (4.9) we may evaluate elements of $q H^{*}(G / P)$ as functions on the points of $\mathcal{Y}_{P}$ and $\mathcal{X}_{P}$, or $X_{P}$.

Definition 7.1. Let the totally positive part of $\mathcal{Y}_{P}$ be defined as

$$
\mathcal{Y}_{P,>0}:=\mathcal{Y}_{P}(\mathbb{R}) \cap\left(G / B^{-}\right)_{\geq 0} \cap B^{-} w_{0} B^{-} / B^{-} \text {. }
$$

This automatically lies in $\mathcal{X}_{P}$, so we also set $\mathcal{X}_{P,>0}:=\mathcal{Y}_{P,>0}$. Finally, compatibly with this definition, set $X_{P,>0}:=X_{P}(\mathbb{R}) \cap U_{>0}^{-}$.

We define the Schubert-positive parts of $\mathcal{Y}_{P}, \mathcal{X}_{P}$ and $X_{P}$, also compatibly with the various morphisms between them, by

$$
\begin{aligned}
& \mathcal{Y}_{P,>0}^{S \text { Schub }}:=\left\{x \in \mathcal{Y}_{P}(\mathbb{R}) \mid \sigma_{w}^{P}(x)>0 \text { all } w \in W^{P}\right\}, \\
& \mathcal{X}_{P,>0}^{S c h u b}:=\left\{x \in \mathcal{X}_{P}(\mathbb{R}) \mid \sigma_{w}^{P}(x)>0 \text { all } w \in W^{P}\right\}, \\
& X_{P,>0}^{S \text { Schub }}:=\left\{x \in X_{P}(\mathbb{R}) \mid \sigma_{w}^{P}(x)>0 \text { all } w \in W^{P}\right\} .
\end{aligned}
$$

These are all semi-algebraic subsets of the real points of $\mathcal{Y}_{P}, \mathcal{X}_{P}$ and $X_{P}$, respectively.

Theorem 7.2. (1) The ramified cover $\pi=\pi^{P}=\left(q_{1}^{P}, \ldots, q_{k}^{P}\right): \mathcal{Y}_{P}(\mathbb{C}) \rightarrow \mathbb{C}^{k}$ restricts to a bijection

$$
\pi_{>0}^{P}: \mathcal{Y}_{P,>0}^{S c h u b} \rightarrow \mathbb{R}_{>0}^{k} .
$$

(2) $\mathcal{Y}_{P,>0}^{S c h u b}$ lies in the smooth locus of $\mathcal{Y}_{P}$, and the inverse of the map $\pi_{>0}^{P}$ : $\mathcal{Y}_{P,>0}^{S c h u b} \rightarrow \mathbb{R}_{>0}^{k}$ is analytic.

(3) The two notions of positivity agree. That is,

$$
\begin{gathered}
\mathcal{Y}_{P,>0}^{S c h u b}=\mathcal{Y}_{P,>0}, \\
\text { and also } \mathcal{Y}_{P,>0}^{S c h u b}=\mathcal{X}_{P,>0}^{S c h u b}=\mathcal{X}_{P,>0} \text { and } X_{P,>0}^{S c h u b}=X_{P,>0} .
\end{gathered}
$$

Remark 7.3. We conjecture that all the analogous results to those stated in Theorem 7.2 should hold true in general type. The stabilizer of the principal nilpotent $f$ in that case should have a totally nonnegative part with a cell decomposition coming from the Bruhat decomposition. And there should be an analogous relationship with Schubert bases for quantum cohomology rings $q H^{*}\left(G^{\vee} / P^{\vee}\right)$ of partial flag varieties of the Langlands dual group, via the Peterson variety for general type.

The bianalyticity of $\pi_{>0}^{B}$ is equivalent to the nonvanishing on $X_{B,>0}$ of the quantum Vandermonde function introduced by Kostant 21, Section 9, and 22. This function on $X_{B}$ is expressed as the determinant of a matrix whose entries are alternating sums of minors. There is no obvious reason why it should be nonvanishing on $X_{B,>0}$. But Theorem $7.2(2)$, which is proved by completely different means, implies that the quantum Vandermonde must take either solely positive or negative values on $X_{B,>0}$. We expect that the values will always be positive, but have checked this so far only in very low rank cases.

We now deduce the corollary stated as Proposition 1.2 in the introduction.

Corollary 7.4. Let $X_{\geq 0}$ denote the semi-algebraic subset of $X(\mathbb{R})$ of totally nonnegative unipotent lower-triangular Toeplitz matrices. Then the restriction

$$
\Delta_{\geq 0}: X_{\geq 0} \longrightarrow \mathbb{R}_{\geq 0}^{n-1}
$$

of $\Delta:=\left(\Delta_{1}, \ldots, \Delta_{n-1}\right)$ is a homeomorphism. 
Proof of the corollary. By Theorem 7.2 we have homeomorphisms

$$
\left(q_{1}^{P}, \ldots, q_{k}^{P}\right): X_{P,>0} \rightarrow \mathbb{R}_{>0}^{k},
$$

one for each parabolic $P$. By Lemma 5.1 the $q_{j}^{P}$ 's are related to the $\Delta_{n_{j}}$ 's by a transformation which is continuously invertible over $\mathbb{R}_{>0}^{k}$. By this observation, and since $X_{\geq 0}=\bigsqcup X_{P,>0}$, we have that

$$
\Delta_{\geq 0}: X_{\geq 0} \longrightarrow \mathbb{R}_{\geq 0}^{n-1}
$$

is bijective. So $\Delta_{\geq 0}$ is continuous, bijective, and a homeomorphism onto its image when restricted to any $X_{P,>0}$. Since $\Delta$ is finite it follows that $\Delta_{\geq 0}^{-1}$ is also continuous.

\section{Grassmannians etc.}

The quantum cohomology rings of Grassmannians have been studied much more extensively than those of partial flag varieties; see for example [4, 15, 36, 37]. The Grassmannian case can be regarded as a kind of toy model for this paper. The main results stated in the previous section generalize properties from the Grassmannian case, which were studied by elementary means, basically playing with Schur polynomials, in 35 . We will briefly recall what happens in that case.

(1) Let $\mathcal{V}_{d, n}$ be the transpose of $\bar{X}_{P_{d}}$ (upper-triangular rather than lowertriangular Toeplitz matrices), notation as in [35]. And let $\mathcal{O}_{\text {red }}\left(\mathcal{V}_{d, n}\right)$ be the reduced coordinate ring of $\mathcal{V}_{d, n}$. Then an incarnation of Peterson's theorem says $\mathcal{O}_{\text {red }}\left(\mathcal{V}_{d, n}\right) \cong q H^{*}\left(G / P_{d}\right)$.

(2) The points of $\mathcal{V}_{d, n}$ are those

$$
u=\left(\begin{array}{cccccc}
1 & a_{1} & \cdots & a_{d} & & 0 \\
& 1 & a_{1} & & \ddots & \\
& & \ddots & \ddots & & a_{d} \\
& & & \ddots & a_{1} & \vdots \\
& & & & 1 & a_{1} \\
& & & & & 1
\end{array}\right)
$$

for which

$$
p(x)=x^{d}+a_{1} x^{d-1}+\cdots+a_{d}=\prod_{j=1}^{d}\left(x+z e^{\left(m_{j} \frac{2 \pi i}{n}\right)}\right)
$$

for some $z \in \mathbb{C}$ and integers $0 \leq m_{1}<\cdots<m_{d}<n$. In other words either $u$ is the identity matrix or otherwise the roots of the generating polynomial $p(x)$ are distinct complex numbers $x_{1}, \ldots, x_{d}$ with $x_{1}^{n}=\cdots=x_{d}^{n}$. Write $u=u\left(x_{1}, \ldots, x_{d}\right)$.

(3) Let $u=u\left(x_{1}, \ldots, x_{d}\right)$ as above, and $w_{\lambda}$ the Grassmannian permutation in $W^{P_{d}}$ corresponding to a Young diagram $\lambda$. The image of the Schubert class $\sigma_{w_{\lambda}}^{P_{d}} \in \mathcal{O}_{\text {red }}\left(\mathcal{V}_{d, n}\right)$ is given by

$$
\sigma_{w_{\lambda}}^{P_{d}}(u)=s_{\lambda}\left(x_{1}, \ldots, x_{d}\right),
$$

where $s_{\lambda}$ is the Schur polynomial associated to $\lambda$. 
(4) Let $\zeta=e^{\frac{2 \pi i}{n}}$, and set $u_{\geq 0}(t)=u\left(t \zeta^{-\frac{d-1}{2}}, t \zeta^{-\frac{d-1}{2}+1}, \ldots, t \zeta^{\frac{d-1}{2}}\right)$. Then

$$
u \geq 0: \mathbb{R}_{\geq 0} \stackrel{\sim}{\longrightarrow}\left(\mathcal{V}_{d, n}\right)_{\geq 0} \quad: \quad t \mapsto u \geq 0(t)
$$

is a homeomorphism, where $\left(\mathcal{V}_{d, n}\right)_{\geq 0}$ denotes the totally nonnegative matrices in $\mathcal{V}_{d, n}$.

(5) The values of the Schubert classes on the $u(t)$ are given by a closed (hooklength) formula, which explicitly shows them to be positive for $t>0$.

(6) The quantum parameter $q$ is given by $q\left(u\left(x_{1}, \ldots, x_{n}\right)\right)=(-1)^{d+1} x_{1}^{n}$. In particular, $q\left(u_{\geq 0}(t)\right)=t^{n}$.

From the proof of Theorem 4.2, in particular from inspection of the matrix $u$ introduced in (4.2), we see directly that $\mathcal{V}_{d, n} \cong \mathcal{Y}_{P_{d}}$ via $u \mapsto u w_{P_{d}} B^{-}$. Notice also that (4) and (6) give the parameterization by quantum parameters of Theorem 7.2 in this special case.

Peterson has announced in [33] that all the quantum cohomology rings $q H^{*}(G / P)$ are reduced. To prove this amounts to showing that the element $\sum_{w \in W^{P}} \sigma_{w} \sigma_{P D(w)}$ is a nonzerodivisor in $q H^{*}(G / P)$ (see also [1]). This is because, for example, if $\sigma \in$ $q H^{*}(G / P)$ is nilpotent, then so are all $\mu \sigma$ for $\mu \in q H^{*}(G / P)$, and the corresponding multiplication operators $M_{\mu \sigma}$ on $q H^{*}(G / P)$ have vanishing trace. But computing these traces by Poincaré duality gives $\operatorname{tr}\left(M_{\mu \sigma}\right)=\left\langle\mu \sigma \sum_{w \in W^{P}} \sigma_{w} \sigma_{P D(w)}\right\rangle_{\mathbf{q}}=0$ and therefore $\sigma \cdot\left(\sum_{w \in W^{P}} \sigma_{w} \sigma_{P D(w)}\right)=0$. It is in fact sufficient to show that $\sum_{w \in W^{P}} \sigma_{w} \sigma_{P D(w)}$ is generically nonvanishing on $\mathcal{Y}_{P}$ (see Lemma 10.1).

Apart from the Grassmannian case where everything is very explicit, and the full flag variety case treated in [21, where the Peterson variety $\mathcal{Y}_{B}$ is irreducible, I do not know a proof that $\sum_{w \in W^{P}} \sigma_{w} \sigma_{P D(w)}$ is generically nonvanishing. But with the help of the explicit results above we can prove at least the following lemma which will come in handy later.

Lemma 8.1. The element of $\mathcal{O}\left(\mathcal{Y}_{P}\right)$ defined by $\sigma_{w_{0}^{P}}^{P}$ takes nonzero values on an open dense subset of $\mathcal{Y}_{P}(\mathbb{C})$.

Proof. Since $\mathcal{X}_{P}$ is open dense in $\mathcal{Y}_{P}$, it suffices to show that $\sigma_{w_{0}^{P}}^{P}$ is nonzero on an open dense subset of $\mathcal{X}_{P}(\mathbb{C})$. By Theorem 4.6 we may furthermore replace $\mathcal{X}_{P}$ by $X_{P}$. So let us identify the Schubert classes $\sigma_{w}^{P}$ with rational functions on $\bar{X}_{P}$ and prove that the top one is generically nonvanishing.

Let $P_{m}$ denote the maximal parabolic with $I^{P_{m}}=\{m\}$ and let $C$ be an irreducible component of the closure $\bar{X}_{P}=\bigsqcup_{P^{\prime} \supseteq P} X_{P^{\prime}}$. If $I^{P}=\left\{i_{1}, \ldots, i_{k}\right\}$, then

$$
\left(\Delta_{n_{1}}, \ldots, \Delta_{n_{k}}\right): \bar{X}_{P}(\mathbb{C}) \longrightarrow \mathbb{C}^{k}
$$

is finite, as pullback of the finite map $\left(\Delta_{1}, \ldots, \Delta_{n-1}\right): X(\mathbb{C}) \longrightarrow \mathbb{C}^{n-1}$. Therefore the restriction of $\left(\Delta_{n_{1}}, \ldots, \Delta_{n_{k}}\right)$ to $C$ is surjective and $C$ intersects all of the subvarieties $\bar{X}_{P_{n_{i}}}$ of $\bar{X}_{P}$.

Now in $q H^{*}(G / P)$ we have

$$
\sigma_{w^{P}}^{P}=\sigma_{s_{1} \cdots s_{n_{1}}}^{P} \cdot \sigma_{s_{1} \cdots s_{n_{2}}}^{P} \cdot \cdots \cdot \sigma_{s_{1} \cdots s_{n_{k}}}^{P} .
$$

Let $x \in X_{P}$. Then tracing through Peterson's isomorphisms gives

$$
\sigma_{s_{1} \cdots s_{n_{j}}}^{P}(x)=G_{n_{j}}^{n_{j}}\left(x w_{0} B^{-}\right),
$$

where $G_{n_{j}}^{n_{j}}$ is as in (4.4). This function extends to $X_{P_{n_{j}}} \subset \bar{X}_{P}$ and is seen to be nonvanishing there using the explicit description of $X_{P_{n_{j}}}$ (see (2) above). Since 
any irreducible component of $\bar{X}_{P}$ meets $X_{P_{n_{j}}}$, we have that $\sigma_{s_{1} \cdots s_{n_{j}}}^{P}$ is generically nonzero on $X_{P}$. The same holds therefore for $\sigma_{w^{P}}^{P}$ as the product of the $\sigma_{s_{1} \cdots s_{n_{j}}}^{P}$.

\section{Proof of Theorem $7.2(1)$}

We must first check that $\pi_{>0}^{P}$ actually takes values in $\mathbb{R}_{>0}^{k}$. This follows from the following observation.

Lemma 9.1. Let $P_{n_{j}}$ be the maximal parabolic defined by $I^{P_{n_{j}}}=\left\{n_{j}\right\}$, and set $v=w_{0}^{P_{n_{j}}} \in W^{P_{n_{j}}}$ to be the longest element. Then $v \in W^{P}$ and we have the following relation in $q H^{*}(G / P)$ :

$$
\sigma_{s_{n_{j}}}^{P} \cdot \sigma_{v}^{P}=q_{j}^{P} \sigma_{v \tau_{j, j}}^{P}
$$

where $\tau_{j, j}=s_{n_{j}} \cdots s_{n_{j+1}-1} s_{n_{j}-1} \cdots s_{n_{j-1}+1}$.

Proof. Let $\alpha$ be a positive root such that $\left\langle\alpha, \omega_{n_{j}}^{\vee}>\neq 0\right.$. So $\alpha=\alpha_{h}+\cdots+\alpha_{l}$ for some $h \leq n_{j} \leq l$. By the Chevalley formula, $\sigma_{v s_{\alpha}}$ appears in the expansion of the product only if $\ell\left(v s_{\alpha}\right)=\ell(v)+1$. If $h<n_{j}<l$ then $\ell\left(v s_{\alpha}\right)=\ell(v)+\ell\left(s_{\alpha}\right) \geq \ell(v)+3$. So assume $h=n_{j}$ or $l=n_{j}$. In either of those two cases $\ell\left(v s_{\alpha}\right)=\ell(v)-1$. Thus, the classical contribution to $\sigma_{s_{n_{j}}}^{P} \cdot \sigma_{v}^{P}$ is indeed zero.

Suppose now $\ell\left(v \tau_{h, l}\right)=\ell(v)-\ell\left(\tau_{h, l}\right)$, where $\tau_{h, l}$ is as in Section 3.8. This is equivalent to assuming $\tau_{h, l}^{-1} \in W^{P_{n_{j}}}$. Since

$$
\tau_{h, l}^{-1}=s_{n_{h-1}+1} \cdots s_{n_{l}-1} s_{n_{l+1}-1} \cdots s_{n_{h}}
$$

sends both $\alpha_{n_{h}}$ and $\alpha_{n_{l}}=s_{n_{h}} \cdots s_{n_{l+1}-1}\left(\alpha_{n_{l}-1}\right)$ to negative roots we must have $h=l=j$. So by quantum Chevalley's rule the only possible quantum contribution to the product $\sigma_{s_{n_{j}}}^{P} \cdot \sigma_{v}^{P}$ is $q_{j}^{P} \sigma_{v \tau_{j, j}}^{P}$. It follows by a direct check that this term does indeed appear (as of course it must, since the product cannot be zero by the same arguments as in Lemma 8.1).

Now we would like to show that $\pi_{>0}^{P}$ is actually surjective. For this fix a point $Q \in\left(\mathbb{R}_{>0}\right)^{k}$ and consider its fiber under $\pi=\pi^{P}$. We may regard

$$
R_{Q}:=q H^{*}(G / P) /\left(q_{1}^{P}-Q_{1}, \ldots, q_{k}^{P}-Q_{k}\right)
$$

as the (possibly nonreduced) coordinate ring of $\pi^{-1}(Q)$. Note that $R_{Q}$ is a finitedimensional algebra with basis given by the (image of the) Schubert basis. We will use the same notation $\sigma_{w}^{P}$ for the restriction of a Schubert basis element to $R_{Q}$.

Lemma 9.2. Suppose $\mu \in R_{Q}$ is a nonzero simultaneous eigenvector for all linear operators $R_{Q} \rightarrow R_{Q}$ which are defined by multiplication by elements in $R_{Q}$. Then there exists a point $p \in \pi^{-1}(Q)$ such that (up to a scalar factor)

$$
\mu=\sum_{w \in W^{P}} \sigma_{w}^{P}(p) \sigma_{P D(w)}^{P} .
$$

Proof. Consider the algebra homomorphism

$$
R_{Q} \longrightarrow \mathbb{C}
$$

which takes $\sigma \in R_{Q}$ to its eigenvalue on the eigenvector $\mu$. This defines the $\mathbb{C}$-valued point $p$ in $\pi^{-1}(Q)$. Now let us write $\mu$ in the Schubert basis,

$$
\mu=\sum_{w \in W^{P}} m_{w} \sigma^{P D(w)}, \quad m_{w} \in \mathbb{C} .
$$


For $\sigma \in R_{Q}$, let $\langle\sigma\rangle_{Q} \in \mathbb{C}$ denote the coefficient of $\sigma_{w_{0}^{P}}^{P}$ in the Schubert basis expansion of $\sigma$. Then by quantum Poincaré duality we have

$$
m_{w}=\left\langle\sigma^{w} \cdot \mu\right\rangle_{Q}=\left\langle\sigma^{w}(p) \mu\right\rangle_{Q}=\sigma^{w}(p)\langle\mu\rangle_{Q}=\sigma^{w}(p) m_{1} .
$$

Here $m_{1}$ must be a nonzero scalar factor (since $\mu \neq 0$ ), and the lemma is proved.

We continue the proof of Theorem 7.2 (1) our immediate aim being to find a Schubert positive point $p_{0}$ in the fiber $\pi^{-1}(Q)$. Set

$$
\sigma:=\sum_{w \in W^{P}} \sigma_{w}^{P} \in R_{Q}
$$

Suppose the multiplication operator on $R_{Q}$ defined by multiplication by $\sigma$ is given by the matrix $M_{\sigma}=\left(m_{v, w}\right)_{v, w \in W^{P}}$ with respect to the Schubert basis. That is,

$$
\sigma \cdot \sigma_{v}^{P}=\sum_{w \in W^{P}} m_{v, w} \sigma_{w}^{P} .
$$

Then since $Q \in \mathbb{R}_{>0}^{k}$ and by positivity of the structure constants it follows that $M_{\sigma}$ is a nonnegative matrix. Furthermore let us assume the following lemma (to be proved later).

Lemma 9.3. $M_{\sigma}$ is an indecomposable matrix.

Given the indecomposable nonnegative matrix $M_{\sigma}$, by Perron-Frobenius theory (see e.g. 31, Section 1.4) we know the following.

The matrix $M_{\sigma}$ has a positive eigenvector $\mu$ which is unique up to a scalar (positive meaning it has positive coefficients with respect to the standard basis). Its eigenvalue, called the Perron-Frobenius eigenvalue, is positive, has maximum absolute value among all eigenvalues of $M_{\sigma}$, and has algebraic multiplicity 1 . The eigenvector $\mu$ is unique even in the stronger sense that any nonnegative eigenvector of $M_{\sigma}$ is a multiple of $\mu$.

Suppose $\mu$ is this eigenvector chosen normalized such that $\langle\mu\rangle_{Q}=1$. Then since the eigenspace containing $\mu$ is 1-dimensional, it follows that $\mu$ is a joint eigenvector for all multiplication operators of $R_{Q}$. Therefore by Lemma 9.2 there exists a $p_{0} \in \pi^{-1}(Q)$ such that

$$
\mu=\sum_{w \in W^{P}} \sigma_{w}^{P}\left(p_{0}\right) \sigma_{P D(w)}^{P} .
$$

Positivity of $\mu$ implies that $\sigma_{w}^{P}\left(p_{0}\right) \in \mathbb{R}_{>0}$ for all $w \in W^{P}$. Hence $p_{0} \in \mathcal{Y}_{P,>0}^{\text {Schub }}$. Also the point $p_{0}$ in the fiber with this property is unique.

Therefore we have shown modulo Lemma 9.3 that

$$
\mathcal{Y}_{P,>0}^{S c h u b} \longrightarrow \mathbb{R}_{>0}^{k}
$$

is a bijection. Finally we complete the proof of Theorem $7.2(1)$ by proving the lemma.

Proof of Lemma 9.3 Recall that $\sigma=\sum_{w \in W^{P}} \sigma_{w}$. Suppose indirectly that the matrix $M_{\sigma}$ is reducible. Then there exists a nonempty, proper subset $V \subset W^{P}$ such that the span of $\left\{\sigma_{v} \mid v \in V\right\}$ in $R_{Q}$ is invariant under $M_{\sigma}$. We will derive a contradiction to this statement.

First take any element $v \in V$. Then the top class $\sigma_{w_{0}^{P}}$ occurs in $\sigma \cdot \sigma_{v}$ with coefficient 1 by quantum Poincaré duality. Therefore we have $w_{0}^{P} \in V$. 
Next we deduce that $1 \in V$. Suppose not. Then the coefficient of $\sigma_{1}$ in $\sigma_{w} \cdot \sigma_{w_{0}^{P}}$ must be zero for all $w \in W^{P}$, or equivalently

$$
\left\langle\sigma_{w} \cdot \sigma_{w_{0}^{P}} \cdot \sigma_{w_{0}^{P}}\right\rangle_{Q}=0
$$

for all $w \in W^{P}$. But this also implies $\left\langle\sigma_{w} \cdot \sigma_{w_{0}^{P}} \cdot \sigma_{w_{0}^{P}}\right\rangle_{\mathbf{q}}=0$, since the latter is a nonnegative polynomial in the $q_{i}^{P}$ 's which evaluated at $Q \in \mathbb{R}_{>0}^{k}$ equals 0 . Therefore $\sigma_{w_{0}^{P}} \cdot \sigma_{w_{0}^{P}}=0$ in $q H^{*}(G / P)$ by quantum Poincaré duality. This leads to a contradiction with Lemma 8.1, that the element $\sigma_{w_{0}^{P}}$ is generically nonzero as a function on $\mathcal{Y}_{P}$. 1

So $V$ must contain 1. Since $V$ is a proper subset of $W^{P}$, we can find some $w \notin V$. In particular, $w \neq 1$. It is a straightforward exercise that given $1 \neq w \in W^{P}$ there exist $\alpha \in \Delta_{+}^{P}$ and $v \in W^{P}$ such that

$$
w=v s_{\alpha}, \quad \text { and } \quad \ell(w)=\ell(v)+1 .
$$

Now $\alpha \in \Delta_{+}^{P}$ means there exists $n_{j} \in I^{P}$ such that $\left\langle\alpha, \omega_{n_{j}}^{\vee}\right\rangle \neq 0$. And hence by the (classical) Chevalley formula we have that $\sigma_{s_{n_{j}}} \cdot \sigma_{v}$ has $\sigma_{w}$ as a summand. But if $w \notin V$ this implies that also $v \notin V$, since $\sigma \cdot \sigma_{v}$ would have summand $\sigma_{s_{n_{j}}} \cdot \sigma_{v}$ which has summand $\sigma_{w}$. Note that there are no cancellations with other terms by positivity of the structure constants.

By this process we can find ever smaller elements of $W^{P}$ which do not lie in $V$ until we end up with the identity element, so a contradiction.

\section{Proof of Theorem $7.2(2)$}

We need to show that $\mathcal{Y}_{P,>0}$ lies in the smooth locus of $\mathcal{Y}_{P}$. Consider the map

$$
E=\left[\left(E_{1}^{(k+1)}, \ldots, E_{n}^{(k+1)}\right)\right]: \mathbb{C}^{n} \longrightarrow \mathbb{C}\left[q_{1}, \ldots, q_{k}\right]^{n},
$$

and its evaluation at $Q=\left(Q_{1}, \ldots, Q_{k}\right) \in \mathbb{C}^{k}$,

$$
E_{Q}=\mathrm{ev}_{Q} \circ E: \mathbb{C}^{n} \longrightarrow \mathbb{C}^{n} .
$$

Here the coordinates $\epsilon_{1}, \ldots, \epsilon_{n}$ of the source $\mathbb{C}^{n}$ are the $\sigma_{i}^{(m)}=: \epsilon_{n_{m-1}+i}$. Let

$$
J_{E}:=\operatorname{det}\left(\frac{\partial E_{i}^{(k+1)}}{\partial \epsilon_{j}}\right)_{i, j} \in \mathbb{C}\left[\sigma_{1}^{(1)}, \ldots, \sigma_{n-n_{k}}^{(k+1)}, q_{1}, \ldots, q_{k}\right],
$$

which at $\mathbf{q}=Q$ evaluates to $J_{E_{Q}}=\operatorname{det}\left(\frac{\partial\left(E_{Q}\right)_{i}}{\partial \epsilon_{j}}\right)_{i, j} \in \mathbb{C}\left[\sigma_{1}^{(1)}, \ldots, \sigma_{n-n_{k}}^{(k+1)}\right]$, the Jacobian of $E_{Q}$. Let us also denote by $J_{E}$ and $J_{E_{Q}}$ the classes these functions define via (3.5) in $q H^{*}(G / P)$ and in $R_{Q}=q H^{*}(G / P) /\left(q_{1}-Q_{1}, \ldots, q_{k}-Q_{k}\right)$, respectively.

Note that the zero-fiber of $E_{Q}$ equals $\left(\pi^{P}\right)^{-1}(Q)$, and a point $p \in\left(\pi^{P}\right)^{-1}(Q)$ is a smooth point of $\mathcal{Y}_{P}$ if the $J_{E_{Q}}(p) \neq 0$. The smoothness assertion of Theorem[7.2 (2) follows from the following lemma.

\footnotetext{
${ }^{1}$ W. Fulton and C. Woodward [14] have in fact recently proved that no two Schubert classes in $q H^{*}(G / P)$ ever multiply to zero. This result can also be recovered as a corollary of Theorem 7.2 since the product of two Schubert classes must take positive values on $\mathcal{Y}_{P,>0}$ and hence cannot be zero.
} 
Lemma 10.1. The element $J_{E} \in q H^{*}(G / P)$ is expressed in terms of the Schubert basis by

$$
J_{E}=\sum_{w \in W^{P}} \sigma_{w} \sigma_{P D(w)} .
$$

Proof. The main ingredient for this lemma is a result from [6] or [36]. But we begin by checking the normalization. Following [18] we have $\left\langle J_{E}\right\rangle_{\mathbf{q}}=\left\langle J_{E_{Q}}\right\rangle_{Q}=\left|W^{P}\right|$. In fact, in terms of the Chern roots $J_{E_{0}}$ is expressed explicitly by

$$
J_{E_{0}}=\prod_{\substack{(i, j), \\ \text { some } 1 \leq n_{m}<j \\ 1 \leq m \leq k}}\left(x_{i}-x_{j}\right) \in \mathbb{C}\left[x_{1}, \ldots, x_{n}\right]^{W_{P}} \cong \mathbb{C}\left[\sigma_{1}^{(1)}, \ldots, \sigma_{n-n_{k}}^{(k+1)}\right],
$$

and hence represents the Euler class in $H^{*}(G / P)$. Therefore, $\left\langle J_{E_{0}}\right\rangle_{0}=\int_{G / P} \chi_{G / P}=$ $\left|W^{P}\right|$. But by its degree $\left\langle J_{E}\right\rangle_{\mathbf{q}}=\left\langle J_{E_{Q}}\right\rangle_{Q}$ is a constant, independent of $Q$.

Now given the normalization as above, [36, Proposition 4.1] says that $\operatorname{Res}_{\mathrm{E}_{\mathbf{Q}}}(\tilde{\eta})=$ $\langle\eta\rangle_{Q}$, where $\tilde{\eta} \in \mathcal{O}\left(\mathbb{C}^{n}\right)$ and $\eta \in R_{Q} \cong \mathcal{O}\left(\mathbb{C}^{n}\right) /\left(\left(E_{Q}\right)_{1}, \ldots,\left(E_{Q}\right)_{n}\right)$ is the class represented by $\tilde{\eta}$. Putting this identity together with [36, Lemma 4.3] we obtain the identity

$$
\operatorname{tr}\left(M_{\kappa}\right)=\left\langle\kappa J_{E_{Q}}\right\rangle_{Q}, \quad \kappa \in R_{Q}
$$

where $M_{\kappa}$ is the multiplication operator by $\kappa$ on $R_{Q}$.

On the other hand this trace may be computed from Poincaré duality by

$$
\operatorname{tr}\left(M_{\kappa}\right)=\left\langle\kappa \sum_{w \in W^{P}} \sigma_{w} \sigma_{P D(w)}\right\rangle_{Q} .
$$

Comparing the two expressions for all $Q$ and all $\kappa$ it follows that

$$
J_{E}=\sum_{w \in W^{P}} \sigma_{w} \sigma_{P D(w)}
$$

as required.

It remains to prove that the inverse to $\pi_{>0}^{P}$ is analytic. This follows from the following lemma.

Lemma 10.2. Choose local coordinates $y_{1}, \ldots, y_{k}$ in a neighborhood of $p_{0} \in X_{P,>0}$. The Jacobian $\mathcal{J}=\operatorname{det}\left(\frac{\partial q_{i}^{P}}{\partial y_{j}}\right)$ is nonzero at the point $p_{0}$.

Proof. Let $Q=\pi^{P}\left(p_{0}\right)$. Let $R=q H^{*}(G / P)$ and let $I \subset R$ be the ideal $\left(q_{1}-Q_{1}\right.$, $\left.\ldots, q_{k}-Q_{k}\right)$. The Artinian ring $R_{Q}=R / I$ is isomorphic to the sum of local rings $R_{Q} \cong \bigoplus_{x \in\left(\pi^{P}\right)^{-1}(Q)} R_{x} / I R_{x}$. And for $x=p_{0}$ the local ring $R_{p_{0}} / I R_{p_{0}}$ corresponds in $R_{Q}$ to the Perron-Frobenius eigenspace of the multiplication operator $M_{\sigma}$ from the above proof. Since this is a one-dimensional eigenspace (with algebraic multiplicity one), we have that $\operatorname{dim}\left(R_{p_{0}} / I R_{p_{0}}\right)=1$. Therefore any nonzero element $r \in R_{p_{0}} / I R_{p_{0}}$ has the property $r\left(p_{0}\right) \neq 0$. But the Jacobian $\mathcal{J}$ gives a nontrivial element in $R_{p_{0}} / I R_{p_{0}}$, since its residue at $p_{0}$ with respect to $I$ is nonzero (see e.g. Chapter 5 in [17]). 


\section{The Schubert classes as rational functions on $\mathcal{Y}$}

To compare Schubert-positivity with total positivity we need to make a closer study of the functions defined by the Schubert classes. The following proposition is one of the most striking features of the Peterson variety picture of quantum cohomology. As far as I understand, it can be extracted from Peterson's statements in [33] or [34] on the connection between each of the $q H^{*}(G / P)$ 's and the homology of the loop group $\Omega K$ of the compact real form of $G$. We will give a direct proof here for type $A$.

Proposition 11.1 (D. Peterson). Let $w \in W$ and let $\sigma_{w}^{B}$ be the corresponding Schubert class regarded as a function on $\mathcal{Y}_{B}$. Let $\widetilde{\sigma}_{w}$ be the rational function on the Peterson variety $\mathcal{Y}=\overline{\mathcal{Y}}_{B}$ defined by $\left.\widetilde{\sigma}_{w}\right|_{\mathcal{Y}_{B}}=\sigma_{w}^{B}$. Then $\tilde{\sigma}_{w}$ is regular on $\mathcal{Y}_{P} \subset \mathcal{Y}$ if $w \in W^{P}$. And in that case we have

$$
\left.\tilde{\sigma}_{w}\right|_{\mathcal{Y}_{P}}=\sigma_{w}^{P} \in \mathcal{O}\left(\mathcal{Y}_{P}\right)
$$

Our proof of this proposition uses the following lemma.

Lemma 11.2. Suppose that $j \in I^{P}$ and $j+1 \notin I^{P}$. Then the rational function $q_{j}^{B}\left(G_{i-1}^{j} G_{l-1}^{j-1}-G_{i-2}^{j-1} G_{l}^{j}\right)$ vanishes on $\mathcal{Y}_{P}$.

Proof. Let $g B^{-} \in \mathcal{Y}_{P}$. Then $\left(g \cdot v_{-\omega_{m}} \mid v_{-\omega_{m}}\right) \neq 0$ precisely if $m \in I^{P}$, and in this case

$$
G_{i}^{m}\left(g B^{-}\right)=\frac{\left(g \cdot v_{-\omega_{m}} \mid s_{m-i+1} \cdots s_{m} \cdot v_{-\omega_{m}}\right)}{\left(g \cdot v_{-\omega_{m}} \mid v_{-\omega_{m}}\right)}
$$

is well defined. Also (5.1) implies that $q_{m}^{B}$ is well defined on $\mathcal{Y}_{P}$ whenever $m \in I^{P}$, and is given by

$$
q_{m}^{B}\left(g B^{-}\right)=\frac{\left(g \cdot v_{-\omega_{m-1}} \mid v_{-\omega_{m-1}}\right)\left(g \cdot v_{-\omega_{m+1}} \mid v_{-\omega_{m+1}}\right)}{\left(g \cdot v_{-\omega_{m}} \mid v_{-\omega_{m}}\right)^{2}} .
$$

Therefore we have

$$
\begin{aligned}
q_{j}^{B}\left(G_{i-1}^{j} G_{l-1}^{j-1}-G_{i-2}^{j-1} G_{l}^{j}\right) & =\frac{\left(g \cdot v_{-\omega_{j+1}} \mid v_{-\omega_{j+1}}\right)}{\left(g \cdot v_{-\omega_{j}} \mid v_{-\omega_{j}}\right)^{3}} \\
& \quad\left(\left(g \cdot v_{-\omega_{j}} \mid s_{j-i+2} \cdots s_{j} \cdot v_{-\omega_{j}}\right)\left(g \cdot v_{-\omega_{j-1}}, s_{j-l+1} \cdots s_{j-1} \cdot v_{-\omega_{j-1}}\right)\right. \\
& \left.\quad-\left(g \cdot v_{-\omega_{j}} \mid s_{j-i+2} \cdots s_{j-1} \cdot v_{-\omega_{j-1}}\right)\left(g \cdot v_{-\omega_{j-1}} \mid s_{j-l+1} \cdots s_{j} \cdot v_{-\omega_{j}}\right)\right) .
\end{aligned}
$$

Now $(j+1) \notin I^{P}$ and $j \in I^{P}$ imply that $\left(g \cdot v_{-\omega_{j+1}} \mid v_{-\omega_{j+1}}\right)=0$ while $\left(g \cdot v_{-\omega_{j}} \mid v_{-\omega_{j}}\right) \neq 0$ on $\mathcal{Y}_{P}$. Hence the above expression vanishes on $\mathcal{Y}_{P}$.

Proof of Proposition 11.1. If $w=s_{h-i+1} \cdots s_{h-1} s_{h}$, then we have

$$
\tilde{\sigma}_{s_{h-i+1} \cdots s_{h}}=G_{s_{h-i+1} \cdots s_{h}}=G_{i}^{h}
$$

and the Proposition holds in this case by Theorem 4.2. Let $w \in W^{P}$ and consider the quantum Schubert polynomial $C_{w}^{P}$ written as a linear combination of $(\mathbf{q}, P)$ standard monomials as in Section 3.6. So

$$
C_{P, w}=\sum_{\Lambda \in \mathcal{L}_{P}} m_{\Lambda} E_{P, \Lambda}, \quad m_{\Lambda} \in \mathbb{C} .
$$


In $E_{P, \Lambda}$ replace each factor $E_{P, i}^{(j)}$ with the corresponding rational function $G_{i}^{n_{j}}$ to define $G_{\Lambda}$. Then as a function on $\mathcal{Y}_{P}$,

$$
\sigma_{w}^{P}=\sum_{\Lambda \in \mathcal{L}_{P}} m_{\Lambda} G_{\Lambda} \mid \mathcal{Y}_{P}
$$

We now use the "quantum straightening identity", [11], Lemma 3.5,

$E_{i}^{(j)} E_{l}^{(j)}=E_{i}^{(j+1)} E_{l}^{(j)}-E_{i-1}^{(j)} E_{l+1}^{(j+1)}+E_{i-1}^{(j)} E_{l+1}^{(j)}+q_{j}\left(E_{i-1}^{(j)} E_{l-1}^{(j-1)}-E_{i-2}^{(j-1)} E_{l}^{(j)}\right)$,

to rewrite $\sigma_{w}^{P}$. Note that a factor $E_{i}^{(j)} E_{l}^{(j)}$ may occur in a $(\mathbf{q}, P)$-standard monomial $E_{\Lambda}$ only if $j \in I^{P}$ and $j+1 \notin I^{P}$. If we replace the $E_{i}^{(j)}$ 's by $G_{i}^{n_{j}}$ in the above identity and apply Lemma 11.2 then we get

$$
\left(G_{i}^{n_{j}} G_{l}^{n_{j}}\right)\left|\mathcal{Y}_{P}=\left(G_{i}^{n_{j+1}} G_{l}^{n_{j}}-G_{i-1}^{n_{j}} G_{l+1}^{n_{j+1}}+G_{i-1}^{n_{j}} G_{l+1}^{n_{j}}\right)\right| \mathcal{Y}_{P}
$$

But the function $\sigma_{w}^{B}$ on $\mathcal{Y}_{B}$ (or equivalently the rational function $\tilde{\sigma}_{w} \in \mathcal{K}(\mathcal{Y})$ ) may be obtained from the expression $\sum_{\Lambda \in \mathcal{L}_{P}} m_{\Lambda} G_{\Lambda}$ we had for $\sigma_{w}^{P}$ by repeated substitutions of the kind

$$
G_{i}^{n_{j}} G_{l}^{n_{j}} \longrightarrow G_{i}^{n_{j+1}} G_{l}^{n_{j}}-G_{i-1}^{n_{j}} G_{l+1}^{n_{j+1}}+G_{i-1}^{n_{j}} G_{l+1}^{n_{j}},
$$

until the resulting expression has no more summands with factors of type $G_{i}^{n_{j}} G_{l}^{n_{j}}$. (These transformations correspond to the classical straightening identities which are used to turn the $P$-standard monomial expansion of $c_{w}$ into the $B$-standard monomial one.) But the substitutions (11.1) do not affect the restriction to $\mathcal{Y}_{P}$. So we are done.

Proposition 11.3. For the Grassmannian permutation $w \in W^{P_{m}}$ define the rational function $G_{w}$ on $G / B^{-}$by

$$
G_{w}\left(g B^{-}\right):=\frac{\left(g \cdot v_{-\omega_{m}} \mid w \cdot v_{-\omega_{m}}\right)}{\left(g \cdot v_{-\omega_{m}} \mid v_{-\omega_{m}}\right)} .
$$

Then

$$
G_{w} \mid \mathcal{Y}=\widetilde{\sigma}_{w} \in \mathcal{K}(\mathcal{Y})
$$

Proof. By Proposition 11.1 it suffices to show that $G_{w} \mid \mathcal{Y}_{B}$ coincides with $\sigma_{w}^{B}$. But this follows from A. N. Kirillov's explicit formula for the corresponding quantum Schubert polynomials (see Section 3.7) together with Peterson's Theorem 4.2, and inspection of the matrix $u$ from (4.2) in the case where $P=B$.

Corollary 11.4. (1) If $y \in \mathcal{Y}_{B,>0}$, then for any $i \in I$ and $w \in W^{P_{i}}$ we have $\sigma_{w}^{B}(y)>0$

(2) If $y \in \mathcal{X}_{P,>0}$ then $q_{i}^{P}(y)>0$ for all $i=1 \ldots, k$.

Proof. (1) is an immediate corollary of Proposition 11.3, since for any $g \in U^{+}$, $G_{w}\left(g B^{-}\right)$is a quotient of nonzero minors of $g$. Part (2) follows from Proposition 11.3 along with Theorem 4.6 and Lemma 9.1. 


\section{Proof of Theorem $7.2(3)$}

We begin with a partial converse to Corollary 11.4(1) in the full flag variety case.

Lemma 12.1. Let $y \in \mathcal{Y}_{B}$ be such that $\sigma_{w}^{B}(y)>0$ for all $w \in W$. Then $y \in \mathcal{X}_{B,>0}$. In other words, $\mathcal{Y}_{B,>0}^{S c h u b}=\mathcal{X}_{B,>0}^{S c h u b} \subset \mathcal{X}_{B,>0}$. And therefore also $X_{B,>0}^{\text {Schub }} \subset X_{B,>0}$.

Proof. By Lemma 9.1 we have that $q_{i}^{B}(y)>0$ for all $i=1, \ldots, k$. Therefore $y \in \mathcal{X}_{B}$. Now we may write $y=x w_{0} B^{-}$for some $x \in X_{B}$. It remains to prove that $x \in U_{>0}^{-}$. The positivity of all the quantum parameters $q_{i}^{B}$ implies by (5.1) that $\Delta_{j}(x)>0$ for all $j=1, \ldots, n-1$. Now by Proposition 11.3 the positivity of the $\sigma_{w}^{B}$ for the Grassmannian permutations $w$ of descent $d$ in $W$ implies the positivity of all the $d \times d$ minors with column set $\{1, \ldots, d\}$ and arbitrary row sets. But this suffices to determine that $x$ is totally positive; see e.g. [3].

Proposition 12.2. $X_{B,>0}^{S c h u b}=X_{B,>0}$.

Proof. By Lemma 12.1 we have the following commutative diagram:

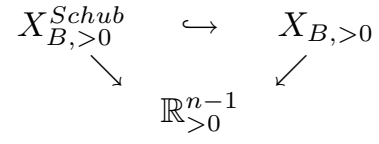

where the top row is clearly an open inclusion and the maps going down are restrictions of $\pi^{B}$. By (1) of Theorem 7.2 , which is already proved, the left-hand map to $\mathbb{R}_{>0}^{n-1}$ is a homeomorphism. It follows from this and elementary point set topology that $X_{B,>0}^{S c h u b}$ must be closed inside $X_{B,>0}$. So it suffices to show that $X_{B,>0}$ is connected.

For an arbitrary element $u \in X$ and $t \in \mathbb{R}$, let

$$
u_{t}:=\left(\begin{array}{ccccc}
1 & & & & \\
t a_{1} & 1 & & & \\
t^{2} a_{2} & t a_{1} & \ddots & & \\
\vdots & & \ddots & 1 & \\
t^{n-1} a_{n-1} & \cdots & t^{2} a_{2} & t a_{1} & 1
\end{array}\right) .
$$

So $u_{0}=\operatorname{Id}$ and $u_{1}=u$, and if $u \in X_{B,>0}$, then so is $u_{t}$ for all positive $t$.

Let $u, u^{\prime} \in X_{B,>0}$ be two arbitrary points. Consider the paths

$$
\begin{array}{ll}
\gamma:[0,1] \rightarrow X_{B,>0}, & \gamma(t)=u u_{t}^{\prime} \\
\gamma^{\prime}:[0,1] \rightarrow X_{B,>0}, & \gamma^{\prime}(t)=u_{t} u^{\prime} .
\end{array}
$$

Note that these paths lie entirely in $X_{B,>0}$ since $X_{B,>0}$ is a semigroup (as the intersection of the group $X$ with the semigroup $U_{>0}^{-}$). Since $\gamma$ and $\gamma^{\prime}$ connect $u$ and $u^{\prime}$, respectively, to $u u^{\prime}$, it follows that $u$ and $u^{\prime}$ lie in the same connected component of $X_{B,>0}$, and we are done.

Corollary 12.3. $\mathcal{Y}_{P,>0}=\mathcal{X}_{P,>0}=\mathcal{X}_{P,>0}^{\text {Schub }}=\mathcal{Y}_{P,>0}^{\text {Schub }}$ and in particular also $X_{P,>0}=X_{P,>0}^{S c h u b}$.

Proof. The identity $\mathcal{X}_{P,>0}^{S c h u b}=\mathcal{Y}_{P,>0}^{S c h u b}$ follows from Lemma 9.1. It remains only to show that $X_{P,>0}=X_{P,>0}^{S c h u b}$. We begin with the inclusion $\subseteq$. Let $X_{\geq 0}=X \cap U_{\geq 0}^{-}$. Then clearly

$$
\overline{X_{B,>0}} \subseteq X_{\geq 0}
$$


is an inclusion of closed subsemigroups of $U^{-}$. We show that this is actually an equality. Suppose $x \in X_{\geq 0}$; then for any $u \in X_{B,>0}$ and $u_{t}$ defined as in (12.1), the curve $t \mapsto x(t)=x u_{t}$ starts at $x(0)=x$ and lies in $X_{B,>0}$ for all $t>0$. Therefore $x \in \overline{X_{B,>0}}$ as desired. As a consequence, using Proposition 12.2, we have

$$
X_{P,>0}=X_{P} \cap \overline{X_{B,>0}}=X_{P} \cap \overline{X_{B,>0}^{\text {Schub }}} .
$$

Now consider the Schubert classes $\sigma_{w}^{P} \in q H^{*}(G / P)$ as functions on $X_{P}$. By Proposition 11.1, $\sigma_{w}^{P}=\left.\tilde{\sigma}_{w}\right|_{X_{P}}$, and $\widetilde{\sigma}_{w}$ takes positive values on $X_{B,>0}^{S c h u b}$. Let us choose $x \in X_{P,>0}$. Then by (12.3) we have also $\sigma_{w}^{P}(x) \geq 0$ for all $w \in W^{P}$. On the other hand $Q:=\pi^{P}(x)=\left(q_{1}^{P}(x), \ldots, q_{k}^{P}(x)\right) \in \mathbb{R}_{>0}^{k}$ by Corollary 11.4 But we have seen in Section 9 that there is only one Schubert nonnegative point in the fiber $\left(\pi^{P}\right)^{-1}(Q)$, and that that one is strictly positive. Thus in fact $\sigma_{w}^{P}(x)>0$ for all $w \in W^{P}$ and $X_{P,>0} \subset X_{P,>0}^{S c h u b}$.

It remains to show that $X_{P,>0} \hookrightarrow X_{P,>0}^{S c h u b}$ is surjective. Consider again the proper map

$$
\Delta=\left(\Delta_{1}, \ldots, \Delta_{n-1}\right): X \rightarrow \mathbb{C}^{n-1}
$$

defined in Section 4.3 Its restriction $\Delta_{\geq 0}=\left(\Delta_{1}, \ldots, \Delta_{n-1}\right): X_{\geq 0} \rightarrow\left(\mathbb{R}_{\geq 0}\right)^{n-1}$ is surjective, since the image must be closed and contain $\Delta_{\geq 0}\left(X_{B,>0}\right)=\mathbb{R}_{>0}^{n-1}$.

From Theorem [7.2(1) along with Lemma 5.1 we know that the further "restriction" of $\Delta$,

$$
\Delta_{>0}^{P}=\left(\Delta_{n_{1}}, \ldots, \Delta_{n_{k}}\right): X_{P,>0}^{\text {Schub }} \rightarrow\left(\mathbb{R}_{>0}\right)^{k},
$$

is bijective. So we have the following diagram:

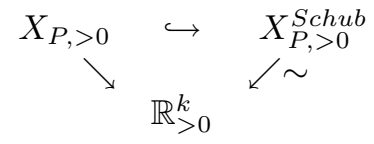

where the downward arrows are given by $\Delta_{>0}^{P}$ and its restriction. By the surjectivity of $\Delta_{>0}$ we also have that the left-hand map is surjective. This implies the desired equality, $X_{P,>0}=X_{P,>0}^{S c h u b}$.

\section{ACKNOWLEDGEMENTS}

Dale Peterson's beautiful results presented by him in a series of lectures at MIT in 1997 were a major source of inspiration and are the foundation for much of this paper. It is a pleasure to thank him here. Some of this work was done during a very enjoyable and fruitful stay at the Erwin Schrödinger Institute in Vienna, and sincere thanks go to Peter Michor for his hospitality. Finally, I would like to thank Bill Fulton for his kind invitation to Michigan, where some of the final writing was done.

\section{REFERENCES}

[1] Lowell Abrams, The quantum Euler class and the quantum cohomology of the Grassmannians, Israel J. Math. 117 (2000), 335-352. MR 2001f:53180

[2] A. Astashkevich and V. Sadov, Quantum cohomology of partial flag manifolds, Comm. Math. Physics 170 (1995), 503-528. MR 96g:58027

[3] Arkady Berenstein, Sergey Fomin, and Andrei Zelevinsky, Parametrizations of canonical bases and totally positive matrices, Adv. Math. 122 (1996), no. 1, 49-149. MR 98j:17008

[4] A. Bertram, Quantum Schubert calculus, Advances in Mathematics 128 (1997), 289-305. MR 98j:14067 
[5] Armand Borel, Sur la cohomologie des espaces fibrés principaux et des espaces homogènes de groupes de Lie compacts, Ann. of Math. (2) 57 (1953), 115-207. MR 14:490e

[6] E. Cattani, A. Dickenstein, and B. Sturmfels, Computing multidimensional residues, Algorithms in algebraic geometry and applications (Santander, 1994), Birkhäuser, Basel, 1996, pp. 135-164. MR 97i:32006

[7] I. Ciocan-Fontanine, Quantum cohomology of flag varieties, Internat. Math. Res. Notices (1995), no. 6, 263-277. MR 96h:14071

[8] _ On quantum cohomology rings of partial flag varieties, Duke Math. J. 98 (1999), no. 3, 485-523. MR 2000d:14058

[9] David A. Cox and Sheldon Katz, Mirror symmetry and algebraic geometry, American Mathematical Society, Providence, RI, 1999. MR 2000d:14048

[10] Albert Edrei, Proof of a conjecture of Schoenberg on the generating function of a totally positive sequence, Canadian J. Math. 5 (1953), 86-94. MR 14:732f

[11] S. Fomin, S. Gelfand, and A. Postnikov, Quantum Schubert polynomials, J. Amer. Math. Soc. 10 (1997), 565-596. MR 98d:14063

[12] S. Fomin and A. Zelevinsky, Total positivity: tests and parametrizations, The Mathematical Intelligencer 22 (2000), 23-33. MR 2001b:15030

[13] W. Fulton and R. Pandharipande, Notes on stable maps and quantum cohomology, Algebraic geometry - Santa Cruz 1995, Amer. Math. Soc., Providence, RI, 1997, pp. 45-96. MR 98m:14025

[14] W. Fulton and C. Woodward, On the quantum product of Schubert classes, Preprint (2001).

[15] D. Gepner, Fusion rings and geometry, Comm. Math. Phys. 141 (1991), 381-411. MR 92j:81271

[16] A. Givental and B. Kim, Quantum cohomology of flag manifolds and Toda lattices, Comm. Math. Phys. 168 (1995), 609-641. MR 96c:58027

[17] Phillip Griffiths and Joseph Harris, Principles of algebraic geometry, John Wiley \& Sons Inc., New York, 1994, Reprint of the 1978 original. MR 95d:14001

[18] B. Kim, Quantum cohomology of partial flag manifolds and a residue formula for their intersection pairings, Internat. Math. Res. Notices (1995), 1-15. MR 96c:58028

[19] Anatol N. Kirillov, Quantum Schubert polynomials and quantum Schur functions, Internat. J. Algebra Comput. 9 (1999), no. 3-4, 385-404, Dedicated to the memory of Marcel-Paul Schützenberger. MR 2001e:05138

[20] Anatol N. Kirillov and Toshiaki Maeno, Quantum double Schubert polynomials, quantum Schubert polynomials and Vafa-Intriligator formula, Discrete Math. 217 (2000), no. 1-3, 191-223, Formal power series and algebraic combinatorics (Vienna, 1997). MR 2001f:05161

[21] B. Kostant, Flag manifold quantum cohomology, the Toda lattice, and the representation with highest weight $\rho$, Selecta Math. (N.S.) 2 (1996), 43-91. MR 97e:17029]

[22] — Quantum cohomology of the flag manifold as an algebra of rational functions on a unipotent algebraic group, Deformation theory and symplectic geometry (Ascona, 1996), Math. Phys. Stud., vol. 20, Kluwer Acad. Publ., Dordrecht, 1997, pp. 157-175. MR 98h:14027

[23] Alain Lascoux and Marcel-Paul Schützenberger, Polynômes de Schubert, C. R. Acad. Sci. Paris Sér. I Math. 294 (1982), no. 13, 447-450. MR 83e:14039

[24] G. Lusztig, Total positivity in reductive groups, Lie theory and geometry: in honor of Bertram Kostant (G. I. Lehrer, ed.), Progress in Mathematics, vol. 123, Birkhäuser, Boston, 1994, pp. 531-568. MR 96m:20071

[25] _ Total positivity and canonical bases, Algebraic groups and Lie groups, Cambridge Univ. Press, Cambridge, 1997, pp. 281-295. MR 2000j:20089

[26] _ Total positivity in partial flag manifolds, Representation Theory 2 (1998), 70-78. MR 2000b:20060

[27] I. G. Macdonald, Notes on Schubert polynomials, LACIM, Montreal, 1991.

[28] _ Symmetric functions and Hall polynomials, 2nd ed., Oxford Univ. Press, 1995. MR 96h:05207

[29] Yuri I. Manin, Frobenius manifolds, quantum cohomology, and moduli spaces, American Mathematical Society, Providence, RI, 1999. MR 2001g:53156

[30] Dusa McDuff and Dietmar Salamon, J-holomorphic curves and quantum cohomology, University Lecture Series, American Mathematical Society, Providence, RI, 1994. MR 95g:58026

[31] Henryk Minc, Nonnegative matrices, John Wiley \& Sons Inc., New York, 1988. MR 89i:15001 
[32] A. Okounkov, On representations of the infinite symmetric group, Zap. Nauchn. Sem. S.Peterburg. Otdel. Mat. Inst. Steklov. (POMI) 240 (1997), Teor. Predst. Din. Sist. Komb. i Algoritm. Metody. 2, 166-228, 294, Translation in J. Math. Sci. (New York) 96 (1999), no. 5, 3550-3589. MR 2000c:20027

[33] D. Peterson, Quantum cohomology of $G / P$, Lecture Course, M.I.T., Spring Term, 1997.

[34] - Quantum cohomology of $G / P$, Séminaire de Mathématiques Supérieures: Representation Theories and Algebraic Geometry, Université de Montreal, Canada, July 28-Aug. 8, 1997 (unpublished lecture notes).

[35] K. Rietsch, Quantum cohomology of Grassmannians and total positivity, Duke Math. J. 113 (2001), no. 3, 521-551.

[36] B. Siebert and G. Tian, On quantum cohomology rings of Fano manifolds and a formula of Vafa and Intriligator, Asian J. Math. 1 (1997), no. 4, 679-695. MR 99d:14060

[37] E. Witten, The Verlinde algebra and cohomology of the Grassmannian, Geometry, topology \& Physics, Conf. Proc. Lecture Notes VI (1995), 357-422. MR 98c:58016

Mathematical Institute, University of Oxford, Oxford, United Kingdom

E-mail address: rietsch@maths.ox.ac.uk

Current address: King's College, University of London, London, United Kingdom

E-mail address: rietsch@mth.kcl.ac.uk 